Submitted to Icarus February 11, 2015; revised June 18, 2015

\title{
Deciphering the Embedded Wave in Saturn's Maxwell Ringlet
}

\author{
Richard G. French ${ }^{1, *}$ \\ Philip D. Nicholson ${ }^{2}$ \\ Mathew M. Hedman ${ }^{3}$ \\ Joseph M. Hahn ${ }^{4}$
}

Colleen A. McGhee-French ${ }^{1}$

Joshua E. Colwell ${ }^{5}$

Essam A. Marouf ${ }^{6}$

Nicole J. Rappaport ${ }^{7}$

1. Department of Astronomy, Wellesley College, Wellesley MA 02481

2. Department of Astronomy, Cornell University, Ithaca NY 14853

3. Department of Physics, University of Idaho, Moscow ID 83844

4. Space Science Institute, Austin TX 78759

5. Department of Physics, University of Central Florida, Orlando FL 32816

6. Department of Electrical Engineering, San Jose State University, San Jose CA 95192

7. JPL, Pasadena CA 91109

* Corresponding author, rfrench@wellesley.edu 


\begin{abstract}
The eccentric Maxwell ringlet in Saturn's C ring is home to a prominent wavelike structure that varies strongly and systematically with true anomaly, as revealed by nearly a decade of high-SNR Cassini occultation observations. Using a simple linear "accordion" model to compensate for the compression and expansion of the ringlet and the wave, we derive a mean optical depth profile for the ringlet and a set of rescaled, backgroundsubtracted radial wave profiles. We use wavelet analysis to identify the wave as a 2-armed trailing spiral, consistent with a density wave driven by an $m=2$ outer Lindblad resonance (OLR), with a pattern speed $\Omega_{p}=1769.17^{\circ} \mathrm{d}^{-1}$ and a corresponding resonance radius $a_{\text {res }}=87,530.0 \mathrm{~km}$. Estimates of the surface mass density of the Maxwell ringlet range from a mean value of $11 \mathrm{~g} \mathrm{~cm}^{-2}$ derived from the self-gravity model to $5-12 \mathrm{~g} \mathrm{~cm}^{-2}$, as inferred from the wave's phase profile and a theoretical dispersion relation. The corresponding opacity is about $0.12 \mathrm{~cm}^{2} \mathrm{~g}^{-1}$, comparable to several plateaus in the outer $\mathrm{C}$ ring (Hedman and Nicholson 2014). A linear density wave model using the derived wave phase profile nicely matches the wave's amplitude, wavelength, and phase in most of our observations, confirming the accuracy of the pattern speed and demonstrating the wave's coherence over a period of 8 years. However, the linear model fails to reproduce the narrow, spike-like structures that are prominent in the observed optical depth profiles. Using a symplectic N-body streamline-based dynamical code (Hahn and Spitale 2013), we simulate analogs of the Maxwell ringlet, modeled as an eccentric ringlet with an embedded wave driven by a fictitious satellite with an OLR located within the ring. The simulations reproduce many of the features of the actual observations, including strongly asymmetric peaks and troughs in the inward-propagating density wave. We argue that the Maxwell ringlet wave is generated by a sectoral normal-mode oscillation inside Saturn with $\ell=m=2$, similar to other planetary internal modes that have been inferred from density waves observed in Saturn's $\mathrm{C}$ ring (Hedman and Nicholson 2013, 2014). Our identification of a third $m=2$ mode associated with Saturnian internal oscillations supports the suggestions of mode splitting by Fuller et al. (2014) and Fuller (2014). The fitted amplitude of the wave, if it is interpreted as driven by the $\ell=m=2 f$-mode, implies a radial amplitude at the 1 bar level of $\sim 50 \mathrm{~cm}$, according to the models of Marley and Porco (1993).
\end{abstract}

Subject Keywords: occultations, planets: rings 


\section{Introduction}

Embedded within a broad gap in the outer $\mathrm{C}$ ring, the eccentric Maxwell ringlet was first identified and its basic properties described from Voyager observations (Esposito et al. 1983; Porco et al. 1984a). With its sharp edges, freely-precessing elliptical shape, and linear width-radius relation, this ringlet has much in common with the uranian $\epsilon$ ring (French et al. 1991). The simple fact that both of these ringlets evidently maintain their elliptical shapes over periods long compared with their differential precession periods led early investigators to propose that each ringlet's own self-gravity counteracted its natural tendency to be circularized as a result of differential apsidal precession due to the planetary oblateness (Goldreich and Tremaine 1979; Porco et al. 1984a). However, more detailed studies of the $\epsilon$ ring's internal optical depth profile cast some doubt on this simple model (Gresh et al. 1989), and later theoretical work emphasized the importance of previouslyneglected collisional interactions in maintaining the shape of such rings (Mosqueira 1996; Chiang and Goldreich 2000).

Since 2005, Cassini observations have greatly increased our store of high-resolution profiles of the Maxwell ringlet. In particular, several sets of radio and stellar occultations have led to greatly-improved kinematic models of the ringlet's edges, with post-fit residuals of $\sim 200 \mathrm{~m}$ (Nicholson et al. 2014b). A by-product of this investigation was the realization that the Maxwell ringlet also hosts a well-developed wavelike structure, which at first sight resembles the numerous density waves in the A ring driven by inner Lindblad resonances (ILRs) with external satellites. This wave was first seen in a single high-resolution Cassini image obtained during Saturn Orbit Insertion (Porco et al. 2005). Unlike the vast majority of these satellite-driven waves, however, the Maxwell wave appears to propagate inwards. This fact alone suggests that it belongs instead in the category of density waves driven at outer Lindblad resonances (OLRs) whose origins are thought to be internal oscillations within Saturn (Marley and Porco 1993; Hedman and Nicholson 2013). However, the fact that this wave, although quite prominent, is embedded within a precessing, eccentric ringlet prevented the straightforward application of the techniques used by Hedman and Nicholson (2013) to analyze a half-dozen similar OLR-type waves in the $\mathrm{C}$ ring.

Here, we conduct a detailed investigation of this wave in order to ascertain its origin and its implications for the dynamics of its host ringlet. For our analysis, we make use of the same extensive set of stellar and radio occultation observations obtained by the Cassini spacecraft and used by Nicholson et al. (2014b). In Section 2, we review the Maxwell ringlet observations used in this work, and in Section 3 the ringlet's average internal optical depth profile is examined, with the goal of updating the classical Goldreich-Tremaine self-gravity model. Next, in Section 4, we use wavelet decomposition to identify the internal wave in the ringlet as an $m=2$ density wave driven at an outer Lindblad resonance, determine its pattern speed, calculate its precise resonance location, and construct a simple linear density wave model as a first approximation to the observed wave. Given the complex morphology of the wave, in Section 5 we move beyond linear models and employ N-body symplectic integrations that successfully reproduce many of the observed properties of the Maxwell

ringlet wave. In Section 6, we compare the observed characteristics of the $m=2$ wave 
with those of other waves believed to be generated by internal $f$-mode oscillations within Saturn, as studied by Hedman and Nicholson (2013) and Hedman and Nicholson (2014). In the final section, we present our conclusions and offer suggestions for future work.

\section{Observations}

Our data set is essentially the same as that used by Nicholson et al. (2014a) and Nicholson et al. (2014b), and consists of $\sim 170$ occultation profiles of Saturn's rings obtained from the Radio Science Subsystem (RSS), Ultraviolet Imaging Spectrometer (UVIS) and Visual and Infrared Imaging Spectrometer (VIMS) experiments on the Cassini orbiter. Of these, 105 profiles span the region of the outer $\mathrm{C}$ ring containing the Maxwell ringlet. The RSS data come primarily from a set of eight nearly radial high quality occultations in 2005/2006, processed to a uniform resolution of $1 \mathrm{~km}$ (French et al. 2010). VIMS data come from $\sim 60$ stellar occultations that sample a wide range of elevation angles, with generally high signal-to-noise ratios and resolutions that range from $300 \mathrm{~m}$ to $1 \mathrm{~km}$. A slightly

smaller set of stellar occultations from the UVIS instrument provide the highest resolution data available - the best UVIS profiles have resolutions as fine as 20-30 m (Colwell et al. 2010). In the present study, we use data from 94 different occultations, interpolated to a sampling interval of $20-100 \mathrm{~m}$.

Due to small errors in the reconstructed trajectory of the spacecraft, and in a few cases to uncertain stellar positions, the raw profiles have systematic radius errors of order $1 \mathrm{~km}$. We correct these errors by applying a small time shift to each data set, determined by the measurements of $\sim 70$ sharp-edged circular or nearly-circular features in the B and $\mathrm{C}$ rings and the Cassini Division, all with well-determined orbits. The corrections are the same as those employed by Nicholson et al. (2014a) and Nicholson et al. (2014b), to which the interested reader is directed for more details.

Figure 1 shows a subset of our data for the Maxwell ringlet, chosen from a series of 17 occultations of the star $\gamma$ Crucis in 2008/2009, and arranged in order of increasing true anomaly (i.e., angle from periapse). As well as obvious variations in the mean radius and width of the ringlet, associated with its eccentric inner and outer edges, this figure shows a strong, wavelike structure that occupies the inner two-thirds of the ringlet. Although the visibility and character of this wave vary with true anomaly, its wavelength and amplitude in all cases decrease inwards.

\section{Average ring properties}

Before embarking on our investigation of the wave embedded within the Maxwell ringlet, we first establish some of the ringlet's global properties, such as its average optical depth profile, and use these to update the self-gravity model first employed by Porco et al. (1984a). Several of these results will also prove useful in the following sections. 


\subsection{Average optical depth profile}

The first step in our analysis is to determine the mean optical depth profile of the Maxwell ringlet. In order to correct for the ringlet's variable width, which ranges from $20.0 \mathrm{~km}$ at periapse to $98.2 \mathrm{~km}$ at apoapse (Nicholson et al. 2014b), we first rescale each profile to a uniform mean width of $59.1 \mathrm{~km}$. This amounts to removing the eccentricity of each streamline within the ring, on the assumption that the eccentricity varies linearly from the inner edge, where $a e=18.9 \mathrm{~km}$, to the outer edge, where $a e=58.0 \mathrm{~km}$; the mean eccentricity of the ringlet is $a \bar{e}=38.5 \mathrm{~km}$ (all orbital parameters are taken from Tables 2 and 5 of Nicholson et al. (2014b)). The resulting scaled radii approximate the semi-major axes of the ring's internal streamlines, which we measure relative to the ringlet's mean semimajor axis, $\bar{a}=87,509.8 \mathrm{~km}$ and denote $\Delta a$. Measured edges are used whenever available; in a few cases (e.g., when short data gaps in the VIMS data fall at the edge of the ringlet) we use the predicted edge radius based on our published orbit model. The raw data are first interpolated onto a uniform 1000-point grid from edge to edge, and then stretched or compressed to the mean ringlet width of $59.1 \mathrm{~km}$.

In rescaling the ringlet's radial profile, we must also adjust the measured optical depths. For the RSS data, we assume an extinction efficiency of 2, due to the separation of the Doppler-shifted scattered signal from the coherent directly transmitted signal (Marouf et al. 1983), and for comparison with the stellar occultation results, we divide the observed RSS optical depths by two. On the reasonable assumption that the total amount of material in the ring per unit length in the azimuthal direction is constant, and on the (less-certain) assumption that surface mass density is proportional to optical depth, we might expect that the average optical depth of the ring varies in inverse proportion to its radial width. We can test this hypothesis by plotting the radially-integrated normal optical depth $A$ (i.e., the equivalent depth, as defined by Elliot et al. (1984)) of the ring vs true anomaly, as in

Fig. 2. For this purpose, we have divided the occultation profiles into groups, denoted by a subjective quality index QI. The 16 highest quality data are assigned QI $=1$; a second tier of 36 profiles with slightly lower SNR but very clear wave structure are assigned QI $=2 ; 42$ profiles with average SNR or somewhat noisy wave structure are assigned QI = 3; and 38 profiles with poor SNR are assigned QI $=4$. Fifteen very poor profiles are assigned $\mathrm{QI}=5$, and are not used in subsequent analyses. Restricting our attention for the moment to QI $=1$ profiles, which are the least likely to suffer from saturation even in the most opaque regions, we find that $A$ is indeed independent of true anomaly, with a mean value $\bar{A}=57.24 \pm 2.30 \mathrm{~km}$. Profiles with QI $=2$ or 3 give systematically lower values, probably because they underestimate the optical depth in the more opaque regions, such as the peaks in the wave. The corresponding mean optical depth of the ringlet $\bar{\tau}=0.968$.

Based on these results, we rescale each measured optical depth profile by a factor of $\Delta r / 59.1 \mathrm{~km}$, where $\Delta r$ is the measured (or if necessary, calculated) radial width of the ringlet. The resulting rescaled profiles for the $16 \mathrm{QI}=1$ data sets are presented in Fig. 3, along with their mean. The mean profile, which we denote by $\tau_{0}(\Delta a)$, has an equivalent depth of $A=56.48 \mathrm{~km}$, very close to the average value of $57.24 \mathrm{~km}$ estimated above. 


\subsection{The self-gravity model revisited}

Before proceeding further, it is desirable to check whether or not the simple linearstretch model described above adequately describes the variations in the ringlet's optical depth profile with true anomaly. A more physically-based model is the self-gravity model originally developed for the uranian $\epsilon$ ring by Goldreich and Tremaine (1979), in which the eccentricity profile across the ring is adjusted in concert with the observed mean surface density profile in such a way as to cancel the tendency of the ringlet's inner and outer edges to precess differentially under the influence of the planet's oblateness. Such a model was first applied to the Maxwell ringlet by Porco et al. (1984a), using Voyager imaging and occultation data.

Starting with the mean optical depth profile $\tau_{0}(\Delta a)$ in Fig. 3, which corresponds to the actual profile $\tau(r)$ at a true anomaly of $90^{\circ}$ (i.e., the profile at quadrature), we follow the prescription of Goldreich and Tremaine (1979) to calculate a radial profile of eccentricity $e(\Delta a)$ that is consistent with the assumed surface mass density profile and uniform apsidal precession of the ringlet under the combined influences of Saturn's second zonal gravity harmonic $J_{2}$ and the ring's own self-gravity. For this calculation, we divide the ringlet into 100 equally-spaced streamlines, each of which has a mass proportional to the local mean optical depth. As boundary conditions, we use the observed eccentricities of the ring's inner and outer edges and its observed precession rate $\dot{\varpi}$ (Nicholson et al. 2014b).

As a check of the validity of the linear-stretch approximation, we compare in Fig. 4 the radial eccentricity profile $a e(\Delta a)$ computed from the self-gravity model with the simple linear model. The agreement is quite good, as shown in the upper panel. The middle panel shows the deviation of the self-gravity eccentricity profile from the linear model, and the bottom panel shows the eccentricity gradient profile, $q=a d e / d a$, compared to the mean value of $\bar{q}=0.66$ for the linear model.

In Fig. 5, we plot the predicted radial surface mass density profile of the Maxwell ringlet for several different values of the true anomaly, based on the eccentricity profile derived from the self-gravity model and the quadrature profile from Fig. 3. For comparison, we also show the predictions of our simple linear-stretch model, scaled to the same edge radii. The agreement between the dynamically-motivated profile and the linear-stretch model is quite good at most longitudes, although the differences become more apparent near pericenter, where the dynamical model is less strongly peaked and more parabolic in shape. (Conversely, the linear-stretch model is flatter than the dynamical model at apocenter.) This comparison should be kept in mind below, when we use the simple linearstretch model to compare wave profiles at different true anomalies.

The self-gravity model also yields an estimate of the ring's total mass $M_{r}=3.6 \times$ $10^{15} \mathrm{~kg}$, equivalent to a sphere of water ice with a radius of $9.9 \mathrm{~km}$. The corresponding mean surface mass density is $\Sigma=11.2 \mathrm{~g} \mathrm{~cm}^{-2}$. This may be compared with the estimate of $\sim 17 \mathrm{~g} \mathrm{~cm}^{-2}$ obtained by Porco et al. (1984a) using the same dynamical model but based on Voyager observations, and to the more approximate estimate of $22 \mathrm{~g} \mathrm{~cm}^{-2}$ obtained by Nicholson et al. (2014b) using only the ringlet's width-radius relation and a much simpler 
2-streamline model. Of course, given the substantial eccentricity gradient of the ringlet, the local surface density varies by a factor of $\sim 5$ from apoapse to periapse.

An apparent failing of the self-gravity model has been the uncomfortably low surface mass densities of $\sim 2 \mathrm{~g} \mathrm{~cm}^{-2}$ that it yields for the narrower uranian $\alpha$ and $\beta$ rings, which appear to be insufficient for these rings to withstand the aerodynamic drag from the extended uranian exosphere and to be inconsistent with the particle size distributions inferred from the Voyager RSS data. (See French et al. (1991) for a more detailed review of these arguments.) These arguments do not, however, apply to the more massive $\epsilon$ ring, where the predicted surface mass density is $25-35 \mathrm{~g} \mathrm{~cm}^{-2}$. We shall find in Section 4 below that the surface density of the Maxwell ringlet inferred from the dispersion relation of the wave is in fact quite consistent with that derived above from the self-gravity model.

A second problem identified with the Goldreich and Tremaine (1979) model is that the derived eccentricity profile is not always consistent with the observed variations in ringlet optical depth profiles with true anomaly. Marouf et al. (1987) compared the Voyager ingress and egress Radio Science occultation profiles of the uranian $\epsilon$ ring with both a simple linear model and the self-gravity model of Goldreich and Tremaine (1979), and found that the former actually provided a better fit to the data. An independent analysis of four stellar occultation profiles for the $\epsilon$ ring obtained by the Voyager Photopolarimeter instrument by Graps et al. (1995) also concluded that the radial profile of $q(a)$, while not quite flat, did not match the predictions of the Goldreich and Tremaine (1979) self-gravity model. (Note that the variation in $q(\Delta a)$ between $\sim 0.5$ and $\sim 0.8$ seen in Fig. 4 is similar to that found for the $\epsilon$ ring using the self-gravity model of Goldreich and Tremaine (1979), and that the

sharp decrease in $q$ near the edges of both the Maxwell and $\epsilon$ rings is probably due to pressure effects at the edges, as discussed by Chiang and Goldreich (2000).) Unfortunately it does not seem possible to apply the same analysis to the Maxwell ringlet data, because the existence of the prominent wave means that the mass interior to a particular semimajor axis is not constant, but is instead dependent on both time and longitude.

Based on the above concerns, and on the empirical results shown in Figs. 3 and 5, we have elected to use the simple linear-stretch model (which amounts to assuming that the dimensionless eccentricity gradient $q$ is constant across the ringlet), rather than the more complex self-gravity model, to rescale the Cassini profiles and remove the variations in the underlying optical depth profile with true anomaly.

\section{Identification of the Maxwell ringlet wave}

\subsection{Background-subtracted optical depth profiles}

Armed with the mean optical depth profile in Fig. 3, we can reprocess the raw occultation profiles such as those in Fig. 1 to highlight the wave. We first linearly rescale each profile, as described above, to a uniform width of $59.1 \mathrm{~km}$ and in optical depth by the factor $\Delta r / 59.1 \mathrm{~km}$. We then subtract the mean optical depth profile of Fig. 3 from the rescaled 
profile, leaving only the variations with respect to the mean profile $\tau_{0}(\Delta a)$. Examples of such rescaled and background-subtracted profiles are shown in Fig. 6, chosen from the list of $\mathrm{QI}=1$ and $\mathrm{QI}=2$ profiles to provide a good sampling of true anomalies.

In this figure, several features of the wave become immediately apparent. First, we note that the wave is visible at all longitudes in the highest-quality data, even within $\sim 10^{\circ}$ of periapse, where the ringlet's average optical depth is quite high. Second, the wavelengths of the first few cycles, which vary considerably between the raw profiles, seem to be approximately independent of true anomaly in the rescaled profiles. Third, the locations of specific maxima and minima change from one profile to another, which indicates that this pattern is not a stationary structure. (We shall see in Section 4.3 that it is in fact a spiral waveform that is propagating inwards.) Fourth, and most striking, we see that for $150^{\circ}<f<320^{\circ}$ the wave shows sharp peaks (maxima) while at other true anomalies the profiles show pronounced troughs (minima). The sharpest peaks and troughs occur at $f \simeq 240^{\circ}$ and $f \simeq 45^{\circ}$, respectively. In the transition regions at around $130-140^{\circ}$ and near $330^{\circ}$ the wave profiles are dominated by neither peaks nor troughs; instead we see waveforms that are highly skewed - almost sawtooth in shape - but reversed in the sense of their asymmetry. Finally, we note that the wave disappears before reaching the inner edge of the ring, likely the result of viscous damping.

It thus appears that the expansion and contraction of the ringlet associated with its eccentricity also results in a similar expansion and contraction of the wave profile. The simple picture that suggests itself is that of the pleats of an accordion expanding and contracting with the motion of the end plates. However, the systematic change from sharplypeaked minima to maxima is unexpected, and not so easily explained by such a simple model.

We have attempted to quantify the striking variation in waveform seen in Fig. 6 by measuring the amplitudes of the highest or lowest peaks in the central part of each wave profile. For each rescaled and background-subtracted profile with QI $=3$ or better, we identify the point within the range $-6 \mathrm{~km}<\Delta a<+6 \mathrm{~km}$ with the greatest deviation from zero. We then fit a gaussian profile to this peak (either positive or negative) and record its amplitude and sign. In Fig. 7, we plot the resulting signed amplitudes vs true anomaly, along with a third-order Fourier fit to guide the eye. The smoothed amplitudes range from $d \tau=-1.3$ at $f=40^{\circ}$ to $d \tau=+0.9$ at $270^{\circ}$, with zero-crossings at $f \simeq 160^{\circ}$ and $\simeq 330^{\circ}$. Note that the former crossing is much more gradual than the latter, in agreement with the varying waveforms in Fig. 6.

\subsection{Wavelet analysis}

The next step in our analysis is to characterize the variation in wave phase with longitude and time, in order to establish its azimuthal wavenumber $m$ and its angular rotation rate, or pattern speed $\Omega_{p}$. For this we employ a variant of the wavelet technique introduced by Hedman and Nicholson (2013), in which the phase difference between pairs of 
background-subtracted and radially scaled optical depth profiles is measured and compared with the predicted value of $\delta \phi$ :

$$
\delta \phi_{\text {pred }}=|m|\left(\delta \lambda-\Omega_{p} \delta t\right)
$$

where $\delta \lambda$ is the difference in inertial longitudes between the two occultation cuts and $\delta t$ is the corresponding difference in observation times. An example of the wavelet decomposition of a Maxwell ringlet profile is shown in Fig. 8. The top panel shows the mean optical depth profile as a function of normalized radius $\Delta a$. The middle panel shows a smoothed, background-subtracted profile whose periodic waveform is decomposed into a 2-D wavelet spectrum in the lower panel. The contours show the wavelet power as a function of radial wavelength (left axis), wavenumber $k$ (right axis), and radius $\Delta a$. The black line in the lower panel traces the ridge-line of the contours, illustrating that the wavelength of strongest wavelet power decreases inward, as expected for an inward-propagating density wave.

For each occultation profile, we use a similar complex wavelet transform $W(k, \Delta a)$ to establish the phase $\phi(k, r)=\tan ^{-1}\left(W_{I} / W_{R}\right)$, where $W_{R}$ and $W_{I}$ are the real and imaginary parts of the transform and $k$ is the radial wavenumber. An average of the phase is computed at each radial location $\Delta a$, weighted by the total wavelet power, $P(k, \Delta a)=W_{R}^{2}+W_{I}^{2}$, to produce the monotonically-varying phase profile $\bar{\phi}(\Delta a)$. The final step is to compute the phase difference $\delta \phi_{\text {obs }}$ between two background-subtracted, normalized, and radially-scaled observations as an average of $\delta \bar{\phi}(\Delta a)$, weighted by the mean power profile $\bar{P}(\Delta a)$. (See Hedman and Nicholson (2013) for more details and examples of this procedure.)

The strength of the difference technique is that, while the mean phase $\bar{\phi}(\Delta a)$ varies rapidly with radius through the wave, the phase difference between two independent radial profiles of the same wave should be constant, and depend only on $\delta \lambda$ and $\delta t$.

\subsection{Wave pattern speed}

Under the assumption that the wave is driven by either an inner or outer Lindblad resonance, the local pattern speed is given by the expression (Hedman and Nicholson 2013):

$$
m \Omega_{p}=(m-1) n+\dot{\varpi}
$$

where $m$ is positive for an ILR and negative for an OLR. Here, $n$ is the local keplerian mean motion and $\dot{\varpi}$ is the apsidal precession rate due to Saturn's zonal gravity harmonics. For each trial value of $m$, and for a series of values of $\Omega_{p}$ in the neighborhood (i.e., within $10 \%$ ) of the expected rate given by this equation, we calculate the predicted phase differences $\delta \phi$ for all possible pairs of occultations and compare these with the observed phase differences. Carrying out this prescription, we find a significant minimum in the RMS phase residuals

$\delta \phi_{\text {obs }}-\delta \phi_{\text {pred }}$ only for an OLR with $m=-2$. (All values of $m$ between -10 and +10 were tested.)

Figure 9 shows the detection of the $m=-2$ wave signature, in the form of a plot of RMS phase residual vs pattern speed. For this calculation we have used a total of 1059 
pairs of VIMS and UVIS profiles of QI $=3$ or better, and with separations in time of less than 300 days. $^{1}$ A pronounced minimum occurs for $\Omega_{p}=1769.17^{\circ} \mathrm{d}^{-1}$, very close to the expected value given by Eq. (2), assuming the gravitational harmonic values given in Jacobson et al. (2006), with an RMS residual of $29.8^{\circ}$. The corresponding resonant radius $a_{\text {res }}=87,530.0 \mathrm{~km}$, or $\Delta a=+20.2 \mathrm{~km}$. The lower panel of this figure shows the individual phase residuals as a function of time interval $\delta t$, verifying that there are no systematic deviations. This technique of scanning over a range of values for $\Omega_{p}$ is the same as that used by Nicholson et al. (2014a) and Nicholson et al. (2014b) to search for normal modes at the edge of the $\mathrm{B}$ ring, and at various gaps and ringlet edges in the $\mathrm{C}$ ring. The key difference here is that we compare phase differences $\delta \phi$ to their predicted values, rather than absolute values of $\phi$.

Although the above analysis indicates that the wave's pattern speed is consistent with that of an OLR with $m=-2$, it is also desirable to verify that its two-dimensional form matches a 2-armed trailing spiral, as expected for any density wave driven at a Lindblad resonance (Shu 1984). We do this in Fig. 10 by comparing wave profiles of varying phase $\phi$ but acquired at similar true anomalies. We choose a range of true anomaly, $200^{\circ}<f<300^{\circ}$, where the profiles are similar in form and the ring is not too narrow. As the phase (and thus the longitude in a frame rotating at an angular rate $\Omega_{p}$ ) increases, we see that the peaks in optical depth move steadily to smaller radii, consistent with a trailing spiral. And after an increase in $\phi$ of $360^{\circ}$ the whole radial pattern of peaks and troughs wraps around, after slipping exactly one cycle. Since we have assumed that $|m|=2$, this corresponds to a rotation of $180^{\circ}$ in physical space, or a 2 -armed spiral pattern.

For completeness, we argue that the wave is a density wave that perturbs the radial optical depth profile in a spiral pattern, rather than a bending wave that results in corrugations of the ring plane itself, driven for example by an inclination resonance with a satellite. The pattern speed of an inward-propagating density wave due to an OLR is faster than the local mean motion, while the pattern speed of an inward propagating bending wave due to an inner vertical resonance is slower than the mean motion. The high pattern speed of the detected wave is strong evidence that the wave is a spiral density wave. An additional argument against the possibility of this being a bending wave is that the visibility of vertical corrugations in a ring is highly dependent on the viewing geometry, and in particular the contrast in optical depth of the sharp spikes and dips observed in the radial profiles of the rings would be much more muted for occultations with large ring opening angles than for events observed at grazing incidence, and this is not the case.

We thus find that the wave in the Maxwell ringlet, after applying our linear-stretch model to remove the effects of the ringlet's eccentricity, behaves almost exactly as expected for a density wave driven at an OLR with $m=-2$. It is thus likely to be yet another example of a $\mathrm{C}$ ring wave driven by internal $f$-mode oscillations in Saturn. This conclusion is supported by the rapid pattern speed, which is higher than the orbital mean motion of

\footnotetext{
${ }^{1}$ Longer time intervals lead to more precisely-defined minima, but also introduce aliasing problems, resulting in multiple, closely-spaced minima. We have found that 300 days is a good compromise.
} 
any known (or even hypothetical) satellite of Saturn. In fact, Marley and Porco (1993) noted the possibility of a causal connection between the $2 f$ resonance and the Maxwell gap, although their best estimate of the resonant radius was $86,215 \pm 550 \mathrm{~km}$, which is $\sim 1300 \mathrm{~km}$ interior to the gap and its ringlet.

\subsection{Radial variation of wave phase}

In order to construct a kinematic model of the wave, we require a prescription for its phase $\phi$ as a function of radius, longitude and time. Following Hedman and Nicholson (2013), we can write this in the form

$$
\phi(\Delta a, \lambda, t)=|m|\left(\lambda-\Omega_{p} t\right)+\phi_{r}(\Delta a) .
$$

The quantity $\phi_{r}(\Delta a)$ could be obtained from our wavelet analysis, to within an unknown constant $\phi_{0}$, as the mean phase $\bar{\phi}(\Delta a)$ introduced above. A simpler and cleaner procedure, however, is to use one of the high-quality occultation profiles (specifically UVIS $\beta$ Cen105E), rescaled to quadrature as described above, and simply identify the sharp peaks in optical depth. Each successive measured peak corresponds to a difference in $\phi_{r}$ of $360^{\circ}$, and we use a least-squares quadratic-interpolation routine to fill in the intermediate values and derive the full radial phase profile $\phi_{r}(\Delta a)$, as shown in Fig. 11. The extrapolated stationary phase point is at $\Delta a=18.9 \mathrm{~km}$, corresponding to a resonant radius, $a_{\text {res }}=87,528.7 \mathrm{~km}$. This is comfortingly close to the value of $a_{\text {res }}$ inferred from the pattern speed of the wave in Fig. 9, giving us confidence that we have a dynamically self-consistent model for the wave.

It is instructive to compare this empirically-derived phase profile with the asymptotic model for a linear density wave, as given by Shu (1984) (cf. Eq. (5) of Hedman and Nicholson (2013)):

$$
\phi_{r}(\Delta a) \simeq 3(m-1) \frac{M_{S}\left(\bar{a}+\Delta a-a_{\mathrm{res}}\right)^{2}}{4 \pi \Sigma_{0} a_{\mathrm{res}}^{4}},
$$

where $M_{S}$ is the mass of Saturn and $\Sigma_{0}$ is the mean surface density of the ring at quadrature. The red curve in Fig. 11 shows such a parabolic model fitted to our adopted phase profile, where the fit parameters are $\Sigma_{0}$ and the resonance location $a_{\text {res }}$. We find that $\Sigma_{0}=15 \mathrm{~g} \mathrm{~cm}^{-2}$, which is in fair agreement with the estimate obtained in Section 3.2 from the self-gravity model, but the fitted value for $a_{\text {res }}$ is unphysical, as it falls within the first

cycle of the wave. We attribute this to the fact that the analytic model applies to a ring of constant background surface density, whereas the actual mean optical depth profile of the Maxwell ringlet is far from uniform. For this reason, we prefer to use our empirical model for $\phi_{r}(\Delta a)$ rather than the quadratic fit.

We can, however, differentiate the empirical phase model to obtain the radial wavenumber $k(\Delta a)=d \phi_{r} / d \Delta a$, and combine this with the differential form of Eq. (4) to estimate the local surface density profile $\Sigma_{0}(\Delta a)$. We find that $\Sigma_{0}$ steadily decreases from $\sim 12 \mathrm{~g} \mathrm{~cm}^{-2}$ at $\Delta a=+5 \mathrm{~km}$ to $\sim 5 \mathrm{~g} \mathrm{~cm}^{-2}$ at $\Delta a=-20 \mathrm{~km}$, mimicking the decline in mean optical depth $\tau_{0}$ over this range. The corresponding opacity of the ring $\kappa=\tau_{0} / \Sigma_{0}$, remains 
relatively constant at $0.12 \mathrm{~cm}^{2} \mathrm{gm}^{-1}$. This is within the range of values found for several nearby plateaus in the outer C ring of $0.11-0.31 \mathrm{~cm}^{2} \mathrm{gm}^{-1}$ (Hedman and Nicholson 2014).

\subsection{Linear density wave model}

With a model for the wave phase $\phi(\Delta a, \lambda, t)$ from Eq. (3) and a numerical expression for $\phi_{r}(\Delta a)$, we can predict the profile of the density wave in any other (rescaled) occultation profile. For simplicity, we employ the linear wave model of Shu (1984) to write the predicted surface density profile in the form

$$
\Sigma(\Delta a, \lambda, t) / \Sigma_{0}=1-A_{L} \Re\left[F(\xi) e^{i m\left(\Omega_{p} t-\lambda-\phi_{0}\right)}\right] e^{-\left(\xi / \xi_{D}\right)^{3}},
$$

where $\Sigma_{0}$ is the unperturbed background surface density of the ring and the dimensionless distance from resonance $\xi$ is simply related to our radial phase $\phi_{r}$ via $\xi^{2}=\phi_{r}(\Delta a)$. The complex oscillatory function $F(\xi)$ involves a Fresnel integral, which must be evaluated numerically. $A_{L}$ is the dimensionless amplitude of the wave and the parameter $\xi_{D}$ is an effective damping length. Eyeball fits of this model to several of the Cassini profiles in Fig. 6 show that a reasonable fit is obtained with the parameters $A_{L}=0.04, \xi_{D}=6$ and a phase offset $\phi_{0}=300^{\circ}$. Linear density wave theory provides estimates of the ring kinematic viscosity $\nu$ and the rms random velocity $c$ from $\xi_{D}$ and the mean ringlet optical depth (cf. Eqs. 7 and 8, and footnote 1, of Tiscareno et al. (2007)). We find $\nu \sim 17$ Stokes $\left(\mathrm{cm}^{2} \mathrm{~s}^{-1}\right)$ and $c \sim 0.13 \mathrm{~cm} \mathrm{~s}^{-1}$, although these values should be regarded as rough estimates only, since $\nu$ depends quite sensitively on $\xi_{D}$, being proportional to $\xi_{D}^{-3}$, whereas $c \propto \xi_{D}^{-3 / 2}$. Recall, too, that the mean optical depth of the Maxwell ringlet increases by a factor of five from apoapse to periapse, further complicating this simple picture.

Combining this model for a linear wave with the mean background optical depth profile $\tau_{0}$ from Fig. 3, we have our final model for the ringlet's rescaled optical depth profile

$$
\tau(\Delta a, \lambda, t)=\tau_{0}(\Delta a) \Sigma(\Delta a, \lambda, t) / \Sigma_{0} .
$$

A comparison of the same 16 high-quality, rescaled wave profiles shown in Fig. 6 with the linear density wave model is presented in Fig. 12. For each occultation we plot three curves: the rescaled optical depth profile in red, the linear wave model in blue, and the background

optical depth profile in green (the latter is the same for all panels.) Comparing the three curves, we make the following points:

1. In most cases, the phase of the model wave matches the observed profile quite well, indicating that our value for the pattern speed is accurate and that the wave has been behaving coherently over a period of 8 years. The only exceptions are near the transition values of $f \sim 130^{\circ}$ and $330^{\circ}$, where the agreement is quite poor.

2. The consistency of the phase match extends well out into the tail of the wave, implying that our radial phase function $\phi_{r}(r)$ (which was derived from a single profile) performs well as a universal model. 
3. Although the model matches the wave's amplitude reasonably well at most longitudes, it does not predict either the strong peaks around $f \sim 270^{\circ}$ or the deep minima around $f \sim 40^{\circ}$.

4. The shape and level of the individual scaled profiles is quite similar to the mean optical depth profile, implying that our simple linear-stretch model describes the actual $\tau$ profiles quite well. The largest deviations occur near periapse $\left(f \simeq 0^{\circ}\right)$, where the linear model overestimates the actual optical depth near the centerline of the ringlet. This is consistent with Fig. 5, where the self-gravity model predicts a less centrally peaked profile near pericenter than does the linear model.

To complete our discussion of the linear density wave model, we present in Fig. 13 a pseudo-image of the Maxwell ringlet with its embedded wave. For this image, we have combined the background mean optical depth profile, linearly stretched as function of true anomaly, with the linear wave model discussed above. The relative widths of the ringlet at periapse and apoapse are accurate, but the absolute widths as well as the ringlet's mean eccentricity have been greatly exaggerated. Higher intensity indicates higher optical depth. The wave is seen propagating inwards, first growing in amplitude and then gradually damping away. Although this is only a snapshot of the ring, one must imagine that this structure is evolving on several timescales: the spiral wave rotates at a rate of $1769^{\circ} \mathrm{d}^{-1}$, while the eccentric ring precesses under the influence of Saturn's $J_{2}$ at a rate of $14.69^{\circ} \mathrm{d}^{-1}$ (period $=24.5$ days). As the wave rotates, its radial scale is alternately compressed and expanded every $4.88 \mathrm{hrs}$, no doubt dissipating significant amounts of energy in inelastic collisions.

\section{N-body simulations of the Maxwell ringlet wave}

Thus far, we have securely established several important characteristics of the Maxwell ringlet density wave, foremost its identity as an inward-propagating $m=2$ OLR with a welldetermined pattern speed, resonance radius, and phase profile $\phi_{r}(\Delta a)$, assuming a simple "accordion" scaling of the internal radial structure to compensate for the substantial width

variations of the ring. A very simple linear density wave model is successful in predicting the phase of the wave for any given occultation observation, matching reasonably well the locations of the wave crests and troughs of the internal wave. However, the actual detailed morphology of the peaks and troughs is not what a linear model would predict. In order to explore the underlying dynamics of the wave more realistically, in this section we present physically-based N-body simulations of the Maxwell ringlet and its internal wave, using an integrator capable of simulating a strong spiral density wave as it propagates in a narrow, eccentric ringlet. 


\subsection{Description of the N-body integrator}

It is a considerable computational challenge to model the dynamics of an optically thick, eccentric ringlet with a large eccentricity gradient and a forced internal wave, using traditional N-body codes. For the Maxwell ringlet, important length scales span the sub$\mathrm{km}$ wavelength of the density wave near periapse to the $20-100 \mathrm{~km}$ variation in ring width from periapse to apoapse. The relevant time scales include the very rapid pattern speed $\left(\sim 1769^{\circ}\right.$ day $\left.^{-1}\right)$, slow precession rate $\left(\sim 14.69^{\circ}\right.$ day $\left.^{-1}\right)$, and the rather long interval for the density wave to propagate from its resonance radius to the inner edge of the ring $(\sim 6$ years, or $\sim 5000$ orbital periods, assuming a group velocity of the wave of $4 \mathrm{~km} \mathrm{yr}^{-1}$ and a mean ring width of $60 \mathrm{~km}$ ). A fully realistic N-body treatment would accommodate the requirement for self-gravity to balance differential precession in the presence of a significantly oblate central planet. Standard N-body co-rotating patch codes with periodic boundary conditions, such as that of Rein and Latter (2013), would require a prohibitively large number of particles to handle such a simulation.

Instead, we employ an N-body integrator - epi_int - that was specifically designed to simulate collective phenomena in a dense planetary ring (Hahn and Spitale 2013, henceforth HS13). This code uses the same drift-kick scheme as the symplectic integrators Symba and Mercury that are widely used to study planetary dynamics (Duncan et al. 1998; Chambers 1999). However epi_int differs from a traditional N-body integrator by not using the usual inverse-square law to calculate inter-particle forces; that approach experiences an artificially high degree of particle-particle scattering due to the necessity of employing simulated particles that are significantly more massive than real ring particles. Such artificial scattering tends to wash out the gentler and more orderly ring phenomena characteristic of a spiral density wave, which is of primary interest here. In epi_int, the positions and velocities of simulated ring particles trace the streamlines that represent the ring's global shape. The acceleration felt by a trace particle due to a nearby streamline is approximated as that due to a straight wire of matter, $2 G \lambda / \Delta$, where $G$ is the gravitational constant, $\lambda$ is the streamline's linear mass density and $\Delta$ is the radial distance between the particle and the streamline.

The use of gravitating wires to calculate ring gravity has been widely used in analytic studies of non-linear ring phenomena (Goldreich and Tremaine 1979; Borderies et al. 1983, 1986), and the streamline concept is well tested and readily implemented in an N-body code. A major benefit of this gravity law is that there is no resultant gravitational scattering as two particles drift past each other in longitude; since each particle represents a segment in a long wire of matter, there is no gravitational impulse that would otherwise occur as discrete particles drift past each other in a traditional N-body model. Consequently, gravitational scattering is greatly reduced and only a modest number of particles is needed to represent a ringlet's full $360^{\circ}$ extent, usually a few thousand.

We note at this point that the surface mass density variations predicted by the linear density wave model (Eq. 5) arise from variations in the distance between streamlines due to trends in the orbital eccentricity and pericenter with semimajor axis. In general, the 
local surface mass density will be inversely proportional to $1-q \cos (\lambda-\varpi+\gamma)$, where the parameters $q$ and $\gamma$ quantify the gradients in the orbital eccentricity and pericenter position $(q \cos \gamma=d(a e) / d a$ and $q \sin \gamma=-a e(d \varpi) / d a$, Borderies et al. (1983)). Note that $\gamma$ should not be confused with the phase of the density wave $\phi$. For typical density waves, the only source of nonzero eccentricities is the wave itself, and so the shapes of the density variations are determined by the strength of the resonant perturbations as well as the average surface mass density and viscosity of the ring. However, in this case we have a wave that is superimposed on top of an eccentric ringlet with its own significant eccentricity gradient, as well as organized pericenter locations that can themselves produce azimuthal variations in the ring's average surface density (Borderies et al. 1983). These background trends in the ring particles' orbital elements cannot change the basic symmetry or the pattern speed of the density wave, but they will introduce extra terms into the expression for the local surface density that can distort the detailed shape of the profiles. Such interactions between the density wave and the ringlet's eccentricity gradient are challenging to explore analytically, an additional motivation to employ a numerical streamline model.

The ring's surface density $\Sigma$ is readily calculated from the density of streamlines, and the N-body epi_int code uses this information to compute the additional forces associated with particle-particle collisions. The integrator assumes that the ring particles are an innumerable swarm of colliding particles. Collisions occurring among particles residing in adjacent parcels in the ring will communicate linear momentum between those parcels, and differences in those rates cause those parcels' trajectories to evolve. The rate at which collisions transfer momentum is controlled by the particle swarm's pressure which, for a flattened 2D cloud of colliding particles, is $p=c^{2} \Sigma$, where $c$ is the dispersion velocity of the particles, chosen so that the mean value of the Toomre stability parameter $Q=c \kappa / \pi G \Sigma$ is equal to 2 (here, $\kappa$ is the epicyclic frequency; note that gravitational stability requires $Q>1$ and the presence of a density wave requires that $Q$ not be larger than a few or so). The net acceleration on any simulated N-body particle due to collisions with particles in adjacent streamlines is thus $A_{p}=-(\partial p / \partial r) / \Sigma$. The model also accounts for the large pressure drop that occurs at a ring's sharp edge: in that case the particle only feels a pressure force from the adjacent streamline, and the acceleration is $A_{p}= \pm p / \lambda$ with the plus (minus) used at the ring's outer (inner) edge.

Collisions among particles in a differentially-rotating ring also transmit angular momentum radially through the ring with flux $F_{\nu}=-\nu_{s} \Sigma r^{2}(\partial \Omega / \partial r)$, where $\nu_{s}$ is the kinematic shear viscosity and $\Omega$ the angular velocity (Borderies et al. 1982). The tangential acceleration that is associated with this radial angular momentum flux is obtained from the differential torque that is exerted across adjacent streamlines, $A_{\nu, \theta}=-\left(\partial F_{\nu} / \partial r\right) / r \Sigma$ in the ring's interior and $A_{\nu, \theta}= \pm F_{\nu} / \lambda r$ at the ring's inner or outer edge, as described in detail in HS13. 


\subsection{N-body models of the Maxwell ringlet}

In principle, the HS13 code is capable of realistically modeling the Maxwell ringlet's eccentric shape, mean optical depth profile at quadrature, the differential precession due to the $J_{2}$ component of Saturn's oblateness, and the response of the ring to a fictitious prograde interior satellite with an $m=2$ OLR located within the ringlet at the inferred resonance radius. In practice, the strong eccentricity gradient and substantial planet-induced differential precession across the ringlet result in streamline crossings that invariably terminate such potentially realistic simulations. After considerable experimentation, we simplified the model's physical parameters in a way that retains the key qualitative characteristics of the Maxwell ringlet and its internal wave, while relaxing other constraints that were not central to our purpose. We suppressed the differential precession due to Saturn by setting $J_{2}=0$, which greatly reduced (but did not completely eliminate) the misalignment of the apses of the inner and outer edges of the ringlet during the integrations. This enabled us to simulate the response of an eccentric ring to resonant forcing, at the expense of exploring the dynamics of ring confinement and apse alignment about an oblate planet. We also reduced the eccentricity gradient across the ring so that the width varied by a factor of about 2 from periapse to apoapse, compared to the actual value of $\sim 5$. Finally, to mimic whatever process prevents the ring from spreading radially, epi_int applies fictitious torques to the ringlet's innermost and outermost streamlines to counterbalance the viscous torques they experience from the ring's interior.

In our exploratory simulations, we varied the assumed mean surface mass density of the ringlet $\Sigma_{0}$ from 5 to $40 \mathrm{~g} \mathrm{~cm}^{-2}$, covering the range of results from the self-gravity analyses described in Section 3.2. The lower surface densities resulted in density waves with relatively short wavelengths and about a dozen wave crests spanning the ringlet, roughly comparable to the observed structure of the Maxwell ringlet. However, these simulations were slow to evolve because of the low group velocity of the satellite-induced wave, and streamline crossing terminated the integrations before the wave had propagated from the resonance radius to the inner regions of the ringlet. The larger surface densities resulted in more rapidly-evolving waves, but with fewer wave crests than the actual Maxwell ringlet. We varied the mass of the fictitious interior satellite from $10^{-11}$ to $10^{-9} M_{S}$, with smaller values resulting in relatively weak, quasi-linear density waves and the larger values resulting in very strongly non-linear waves that quickly resulted in streamline crossing. In other experiments, we compared simulations that included and excluded the pressure forces associated with collisions, and found little difference in the results, although including collisional pressure tended to delay the inevitable streamline crossing that terminated the calculations. We assumed a Toomre parameter $Q=2$ for the results shown here. The corresponding dispersion velocity is $c=1.75 \times 10^{-2} \mathrm{~cm} \mathrm{~s}^{-1}$, significantly smaller than the estimate provided by the linear density wave result found previously in Section 4 . (We experimented with dispersion velocities of 0.175 and $1.75 \mathrm{~cm} \mathrm{~s}^{-1}$, but the wave pattern was strongly deformed and streamline crossing rapidly terminated those simulations.) Finally, we explored a range of assumed shear and bulk viscosities between 0 and 2000 Stokes, with the expected result that wave damping was stronger with higher viscosities. 
Figure 14 shows representative results of the late stage of evolution for an epi_int simulation of a Maxwell-like ringlet with a forced $m=2$ OLR. For this run, we used 200 streamlines with 64 particles per streamline, for a total of 12,800 particles; nearly identical results were obtained with 400 streamlines and 128 particles per streamline $(51,200$ particles). This 2-D polar projection of the ring shows the ringlet's surface mass density just prior to termination of the simulation resulting from streamline crossing near the most opaque part of the ringlet. (The surface density is obtained by interpolation of particle positions along streamlines to determine the linear mass density of each streamline, and then by interpolation of the radial separation of adjacent streamlines to determine the local mass density.) The simulation was begun with an initially uniform radial optical depth profile, and the pronounced wave structure emerged over the course of about 4800 orbital periods ( 1450 days), shortly after which streamline crossing terminated the integration. For this simulation, we included the pressure effects associated with particle collisions, as described above, and assumed values for the bulk and shear viscosities of 100 Stokes, a bit larger than the value found from the linear density wave model in Section 4 . We set $\Sigma_{0}=10 \mathrm{~g} \mathrm{~cm}^{-2}$, comparable to estimates provided by the self-gravity analysis and as inferred from the wave's phase profile and a theoretical dispersion relation. The mass of the fictitious resonant satellite producing the internal wave was $1.0 \times 10^{-10} M_{S}$, about one-third of the mass of Prometheus or eight times that of Atlas. Because the wave may not have reached its steady-state amplitude before the completion of the simulation, this mass is best thought of as an approximate upper limit to that needed to generate a wave of this amplitude.

The variation in radial structure with ring longitude (or true anomaly) is more easily seen in Fig. 15, which shows radial surface density profiles from our simulation sampled every 10 degrees in true anomaly from the polar projection in Fig. 14. The raw profiles are plotted relative to true radius in the left panel (without subtracting a mean background radial profile), and at right we have rescaled the profile widths to a constant value, while at the same time scaling the surface density to preserve the radially integrated optical depth for each individual profile. Note that at any given radius two complete wave cycles are traversed as we move through $360^{\circ}$ in longitude, as expected for an $m=2$ wave. In this simulation, as in the real ringlet, the waveform changes from having strong peaks in density near $f=300^{\circ}$ to sharper troughs around $f=40^{\circ}$, with a broad transition region around $f \sim 160^{\circ}$ (cf. Fig. 7). Although the profiles are by no means identical to those in Fig. 6, the similarity suggests that it is the interaction of the density wave with the eccentricity gradient across the ringlet that is responsible for the unexpected changes in the wave profile with true anomaly. The epi_int simulation of the Maxwell ringlet shows a shift in periapse longitude of a few degrees from the inner to the outer edge. The latter manifests itself as an offset in the minimum ringlet width by $\sim 20^{\circ}$ from $f=0^{\circ}$. Such effects are predicted by more sophisticated versions of the self-gravity model (Borderies et al. 1983). However, in our model we have set the planet's $J_{2}$ (and thus the ringlet's precession rate) to zero, so one should be wary of drawing any direct analogies. Indeed, this shift may be the manifestation of a very long period libration that has not been completely sampled over the relatively short duration of the N-body simulation. 
Overall, the epi_int simulations of the Maxwell ringlet successfully produce a strong density wave with radial profiles that more closely resemble the actual observations than does our simple linear model. Although the integrations eventually terminate because of streamline crossing, they capture several key characteristics of the actual observations, including both prominent peaks and troughs in optical depth and a realistic variation in wavelength and amplitude across the ringlet. They also provide a rough upper limit to the mass of the fictitious satellite that drives the wave, which we will use in the next section to estimate the nature of the forcing of the wave. Although a detailed dynamical explanation for the radial optical depth variations of the wave remains elusive, some insight can be gained from an examination of the eccentricity and periapse longitudes of the streamlines at the conclusion of the numerical simulations, shown in Figure 16. At the upper left, the eccentricity (scaled by orbital radius) is plotted as a function of semimajor axis. The trend is very close to linear, and the deviations from a linear fit are shown in the lower left panel, where the periodic structure is clearly associated with the peaks and troughs in the density wave seen in the simulated optical depth profiles. The general structure of the eccentricity gradient is almost inverted from that seen in Fig. 4 for the Goldreich and Tremaine (1979) self-gravity model, but since we have set $J_{2}=0$ in the epi_int integrations, we should not expect exact agreement between these two cases. The two panels at right show the corresponding results for the periapse longitude of the streamlines.

These results, while encouraging, still leave room for improvement. As noted previously, our simulations have a substantially smaller average eccentricity gradient than the actual Maxwell ringlet. We have also ignored the effects of differential precession on the overall structure of the ring, and on its variation with true anomaly, though in Fig. 5 we have seen that these effects are quite modest. Finally, we note that there is more detailed structure in the observed radial optical depth profiles than we see in our simulations. It is likely that a more sophisticated treatment of close particle encounters than assumed in epi_int will be required to understand how these structures are formed, and to enable the simulations to continue without resulting in streamline crossing. For example, it may be important to model shocks in a dense particle environment when nearby streamlines are pinched close together as particles approach ring periapse. Nevertheless, the N-body symplectic streamline approach to modeling an eccentric ringlet with an embedded wave holds considerable promise for providing insight into the dynamics of such complex ringlets.

\section{Origin of the Maxwell ringlet wave}

The pattern speed of the wave in the Maxwell ringlet is much greater than the orbital mean motion of any of Saturn's moons, by almost a factor of 3. This, combined with the fact that the wave propagates inwards and is thus almost certainly driven at an outer Lindblad resonance, makes it extremely unlikely that this wave is created by any normal satellite resonance. Instead, it is most likely generated by a resonance with a normal-mode

oscillation inside Saturn. Assuming a nominal model for Saturn's internal structure, Marley and Porco (1993) computed the pattern speeds of several different fundamental sectoral (i.e., 
$m=\ell$ ) normal modes that might produce OLR-type resonances in Saturn's rings.

Planetary internal modes come in three flavors: high-frequency $p$ - or pressure - modes and low-frequency $g$ - or gravity- modes, and the intermediate-frequency $f$ - or fundamental - mode, which partakes of both characters. In a largely convective planet such as Saturn, $g$-modes have zero frequency, but both $f$ and $p$ modes are expected. Each mode is characterized by angular "quantum numbers" $\ell$ and $m$, which specify the total number of nodal circles at the planet's surface, as well as the number of radial nodes, specified by $n$. For $f$-modes, $n=0$, while for $p$-modes, $n=1,2, \ldots$ Coriolis forces lead to rotational splitting of modes with different $m$ values. However, modes with few or no radial nodes, and with no latitudinal nodes (i.e., $f$-modes with $m=\ell$ ) are the ones that couple most strongly to the radial epicyclic motions of the ring particles and thus are most likely to generate density waves. These are the sectoral $f$-modes, which may be parametrized by a single number, $\ell$. Marley and Porco (1993) found that the resonance with the $\ell=2$ sectoral $f$-mode (which they denoted $2 f$ and for which they estimated a pattern speed of $\sim 1810^{\circ} \mathrm{d}^{-1}$ ) was likely to fall in the vicinity of the Maxwell gap, and thus suggested that there could be some causal connection between that resonance and the Maxwell ringlet.

Using Cassini occultation data, Hedman and Nicholson (2013) determined the pattern speeds and $m$-numbers of multiple waves throughout the $\mathrm{C}$ ring, identifying $m=-4,-3$ and -2 waves within the regions where Marley and Porco (1993) had predicted the resonances with the relevant normal modes could reside. This finding supported the idea that normal mode oscillations inside Saturn could produce detectable waves in the rings. However, this analysis also revealed that there were three $m=-3$ waves and two $m=-2$ waves, which was surprising since Marley and Porco (1993) had predicted that each sectoral $f$-mode should produce a single wave. There should thus be only one $m=-2$ wave and one $m=-3$ wave at the appropriate ring locations. Recent investigations of higher-order resonances (Marley 2014) do not yield any obvious candidates for nearby additional resonances, and so the multiple waves for a single $m$-value must reflect some aspect of the planet's internal structure that is not captured in simple fluid models. Indeed, recent work suggests that including a solid core or a stably-stratified layer inside the planet could potentially produce the multiple $m=3$ and $m=2$ modes (Fuller et al. 2014; Fuller 2014). ${ }^{2}$

The two $m=-2$ waves characterized by Hedman and Nicholson (2013) occur at $84,644 \mathrm{~km}$ and $87,189 \mathrm{~km}$, respectively. The latter lies just interior to the Maxwell gap, and so is quite close to the $m=-2$ wave located within the Maxwell ringlet. All three of these $m=-2$ waves lie within the uncertainty range of Lindblad resonances with the $2 f$ normal mode oscillations computed by Marley and Porco (1993), so it is likely that all three are generated by such oscillations inside the planet. Furthermore, by providing the third example of an $m=-2$ structure in the $\mathrm{C}$ ring, our analysis of the Maxwell ringlet wave

\footnotetext{
${ }^{2}$ The presence of solid or subadiabatic regions deep within the planet permits the existence of $g$-modes with nonzero frequencies which, if the frequencies are just right, can overlap with the some of the $f$-modes, leading to multiple eigenmodes of mixed character. The observed modes would thus be neither pure $f$-modes nor pure $g$-modes, but for simplicity we shall refer to them here as $f$-modes.
} 
reveals that there are the same number of $m=2$ and $m=3$ oscillation modes in the planet with sufficient magnitude to produce observable wave signatures in the rings. However, the pattern speeds of the $m=-2$ waves span a much broader range than do the $m=-3$ waves, implying that whatever process is responsible for splitting these modes affects the frequency of the $2 f$ mode much more strongly than the $3 f$ mode. The implications of this result for Saturn's interior are still unclear and will require further investigation.

In Fig. 17, we update the summary plot of Hedman and Nicholson (2014) to put the Maxwell ringlet wave in the context of other wave features in the $\mathrm{C}$ ring attributed to either satellite resonances or Saturnian $f$-mode oscillations.

The amplitude of the Maxwell ringlet wave also provides an opportunity to estimate the amplitude of the planetary oscillations. Hedman and Nicholson (2013) did not attempt to estimate the amplitudes of the oscillations required to produce the waves they studied because all of those structures involved opacity variations of order unity and so were probably non-linear. The density variations in the Maxwell ringlet, however, are less extreme and so may provide a more robust estimate of the perturbation amplitude. Our numerical simulations in Section 5 indicate that the perturbations inside the planet are roughly equivalent to a satellite with a mass of $1.0 \times 10^{-10} M_{S}$, or $\sim 6 \times 10^{16} \mathrm{~kg}$. This is about one-third of the mass of Prometheus. Using a similar argument to that of Hedman and Nicholson (2014), who estimated the internal density perturbation needed to produce several waves in the C-ring plateaus that appear to be generated by persistent mass anomalies co-rotating with Saturn's winds, we calculate an average density perturbation for a global mode of $\delta \rho / \bar{\rho} \simeq 4 \times 10^{-10}$.

The permanent mass anomalies inside the planet responsible for the $m=+3$ waves identified by Hedman and Nicholson (2014) are of order $10^{14}-10^{15} \mathrm{~kg}$, or a factor of $50-500$ times smaller than what seems to be required to produce the wave in the Maxwell ringlet. This result is not unreasonable, given that these waves appear to be generated by something tied to Saturn's winds, rather than oscillations in the planet's deep interior.

Another approach to estimating the amplitude of the $2 f$ normal mode is to compare the amplitude of the linear density wave fit in Section 4.5 with that predicted by Marley and Porco (1993), who calculate a normalized surface density amplitude of $6.6 \mathrm{~g} \mathrm{~cm}^{-2}$ for the wave driven by the $\ell=m=2 f$-mode, assuming a maximum radial displacement at the 1-bar level in Saturn of $\Delta r=1 \mathrm{~m}$ (see their Table 1). Scaling this to the amplitude of our linear model - as measured at the peak of the wave at $\xi \simeq 4-$ of $\sim 0.23 \Sigma_{0} \simeq 2.8 \mathrm{~g} \mathrm{~cm}^{-2}$, we estimate a surface amplitude of $\Delta r \simeq 40 \mathrm{~cm}$. This is remarkably close to the amplitude estimated by Marley and Porco (1993), on the assumption that the resonant torque is responsible for maintaining the Maxwell gap. It is also consistent with the $30 \mathrm{~cm}$ amplitude estimated by Marley (1991), on the basis of equipartition of energy between modes and excitation by convective turbulence.

We can also compare the strength of the normal-mode resonance responsible for the Maxwell ringlet wave with that of the satellite resonances associated with the other narrow gaps in the $\mathrm{C}$ ring. Although the precise mechanism whereby any of these gaps - three 
of which have embedded ringlets - is maintained is unclear, we now have a total of four gaps that are located astride resonances. The Colombo gap and Titan ringlet in the inner $\mathrm{C}$ ring are associated with the Titan 1:0 apsidal resonance; the Maxwell gap and ringlet are apparently associated with the $2 f$ internal mode of Saturn; the Bond ringlet's outer edge is perturbed by the Prometheus 2:1 ILR; and the Dawes gap's inner edge appears to be controlled by the Mimas 3:1 ILR. Using satellite resonance strengths tabulated by Nicholson et al. (2014b) (see their Table 10) and the rough estimate provided above for the mass needed to drive the Maxwell ringlet wave, we find relative strengths for these four resonances of 570, 1.0, 2.8 and 12, respectively. On this same scale, the strength of the Mimas 2:1 ILR at the outer edge of the B ring is $\sim 670$ (Nicholson et al. 2014a). (We have assumed here that a mass equal to one-third of Prometheus needed to drive the Maxwell ringlet wave at a first-order $m=-2$ OLR translates into a resonance strength which is $\sim 1 / 3$ that of the Prometheus 2:1 resonance, which is a first-order $m=2$ ILR.) Unfortunately, these relative strengths are not obviously correlated with the corresponding gap widths of 170, 260, 37 and $20 \mathrm{~km}$, respectively; in fact, the widest gap (Maxwell) is associated with the weakest resonance. Clearly, more work is needed to understand the relationship between these gaps and their resonances.

\section{Conclusions and open questions}

High-SNR Cassini occultation observations spanning nearly a decade have provided an extraordinarily detailed view of the radial structure of the eccentric Maxwell ringlet in Saturn's C ring, revealing a prominent wavelike structure that varies strongly and systematically with true anomaly. Using a simple "accordion" model to compensate for the compression and expansion of the ringlet and the wave, we derive a mean optical depth profile for the ringlet and a set of rescaled, background-subtracted radial wave profiles. We use wavelet analysis to identify the wave as a 2-armed trailing spiral, consistent with a density wave driven by an $m=2$ OLR, with $\Omega_{p}=1769.17^{\circ} \mathrm{d}^{-1}$ and a corresponding resonance radius $a_{\text {res }}=87,530.0 \mathrm{~km}$. Using one of the rescaled optical depth profiles, we create a measured wave phase profile across the ring. Estimates of the surface mass density of the Maxwell ringlet range from a mean value of $11 \mathrm{~g} \mathrm{~cm}^{-2}$ derived from the self-gravity model to $5-12 \mathrm{~g} \mathrm{~cm}^{-2}$, as inferred from the wave's phase profile and a theoretical dispersion relation. The corresponding opacity is about $0.12 \mathrm{~cm}^{2} \mathrm{~g}^{-1}$, comparable to several plateaus in the outer $\mathrm{C}$ ring (Hedman and Nicholson 2014).

A linear density wave model that makes use of the above phase profile nicely matches the wave's approximate amplitude, wavelength, and phase in most of our observations, confirming the accuracy of the pattern speed and demonstrating the wave's coherence over a period of 8 years. However, the linear model fails to reproduce the narrow, spike-like structures that are prominent in the observed optical depth profiles.

Making use of epi_int, a symplectic N-body streamline-based dynamical code, we simulate analogs of the Maxwell ringlet, modeled as an eccentric ringlet with an embedded 
wave driven by a fictitious satellite with an OLR located within the ring. The simulations reproduce many of the features of the actual observations, including strong, asymmetric peaks and troughs in the inward-propagating density wave. The observed amplitude of the wave is best matched by an assumed mass for the fictitious satellite of about $1 \times$ $10^{-10} M_{S}$, about one-third the mass of Prometheus. The assumed mean surface density $\Sigma_{0}=10 \mathrm{gm} \mathrm{cm}^{-2}$ matches the predictions of the Goldreich and Tremaine (1979) selfgravity model as well as the observed wave phase profile. The corresponding Saturn density perturbation sufficient to produce the observed wave is about $\delta \rho / \bar{\rho} \simeq 4 \times 10^{-10}$.

This N-body simulation represents a marked improvement over the linear density wave model, but it nevertheless fails to reproduce the detailed trends in the wave's optical depth profile and its variations with true anomaly. It will be necessary to improve the physics of the code to deal more realistically with the highly compressed conditions that currently result in intersecting streamlines, causing the simulations to terminate. An additional important augmentation of the simulations would be to include a physically realistic model for self-gravity or other means of preventing differential precession of the narrow ringlet due to planetary oblateness.

We argue that the Maxwell ringlet wave is generated by a sectoral normal-mode oscillation inside Saturn with $\ell=m=2$, similar to other planetary internal modes that have been inferred from density waves observed in Saturn's C ring (Hedman and Nicholson 2013, 2014). Indeed, an association between the Lindblad resonance associated with this mode and the Maxwell gap was first suggested by Marley and Porco (1993), and more recently by Fuller (2014), who predicted that the wave train in the ringlet would have $m=-2$, as we have confirmed. Whether or not the Maxwell ringlet, or the gap in which it lies, is actually generated by this particular mode of Saturn remains a matter of speculation at this time.

Our identification of a third $m=2$ mode associated with Saturnian internal oscillations supports the suggestions of mode splitting by Fuller et al. (2014) and Fuller (2014), but a deeper understanding of how this comes about will require further study. The fitted amplitude of the wave, if it is interpreted as driven by the $\ell=m=2 f$-mode, implies a radial amplitude at the 1 bar level of $\sim 50 \mathrm{~cm}$, according to the models of Marley and Porco (1993).

We are left with a number of vexing puzzles. How does it happen that the Maxwell ringlet is located exactly at the location of a resonance? Why is the wave so nicely centered in the ringlet? It seems unlikely that this is purely coincidental, and other narrow and sharp-edged ringlets also have internal wavelike structure - as yet unidentified with specific resonances - but we have not yet articulated the possible connections between the formation and permanence of the ringlets and resonantly-produced waves. The rich set of Cassini observations of Saturn's rings will continue to provide a solid basis for such dynamical investigations. 


\section{Acknowledgements}

We gratefully acknowledge support from the NASA Cassini Data Analysis Program, Grant NNX09AE66G, as well as fruitful discussions with Mark Marley, Jim Fuller, and Peter Goldreich. JMH's efforts were supported by the National Science Foundation via Grant No. AST-1313013, and he thanks Byron Tapley for graciously providing office space and the use of the facilities at the University of Texas Center for Space Research (CSR).

\section{REFERENCES}

Baillié, K., Colwell, J. E., Lissauer, J. J., Esposito, L. W., Sremčević, M. 2011. Waves in Cassini UVIS stellar occultations. 2. The C ring. Icarus 216, 292-308.

Borderies, N., Goldreich, P., Tremaine, S. 1982. Sharp edges of planetary rings. Nature 299, 209-211.

Borderies, N., Goldreich, P., Tremaine, S. 1983. The dynamics of elliptical rings. Astron. J. 88, 1560-1568.

Borderies, N., Goldreich, P., Tremaine, S. 1986. Nonlinear density waves in planetary rings. Icarus 68, 522-533.

Chambers, J. E. 1999. A symplectic integration scheme that allows close encounters between massive bodies. Impact of Modern Dynamics in Astronomy 449.

Chiang, E. I., Goldreich, P. 2000. Apse alignment of narrow eccentric planetary rings. Astrophys. J. 540, 1084-1090.

Colwell, J. E., Esposito, L. W., Jerousek, R. G., Sremčević, M., Pettis, D., Bradley, E. T. 2010. Cassini UVIS stellar occultation observations of Saturn's rings. Astron. J. 140, 1569-1578.

Duncan, M. J., Levison, H. F., Lee, M. H. 1998. A multiple time step symplectic algorithm for integrating close encounters. Astron. J. 116, 2067-2077.

Elliot, J. L., French, R. G., Meech, K. J., Elias, J. H. 1984. Structure of the Uranian rings. I - Square-well model and particle-size constraints. Astron. J. 89, 1587-1603.

Esposito, L. W., and 10 colleagues 1983. Eccentric ringlet in the Maxwell gap at 1.45 Saturn radii multi-instrument Voyager observations. Science 222, 57-60.

French, R. G., Nicholson, P. D., Porco, C. C., Marouf, E. A. 1991. Dynamics and structure of the Uranian rings. In: Bergstralh, J. T., Miner, E. D., Matthews, M. S. (Eds.), Uranus. University of Arizona Press, Tucson AZ, 327-409.

French, R. G., Marouf, E. A., Rappaport, N. J., McGhee, C. A. 2010. Occultation observations of Saturn's B ring and Cassini Division. Astron. J. 139, 1649-1667. 
Fuller, J. 2014. Saturn ring seismology: Evidence for stable stratification in the deep interior of Saturn. Icarus 242, 283-296.

Fuller, J., Lai, D., Storch, N. I. 2014. Non-radial oscillations in rotating giant planets with solid cores: Application to Saturn and its rings. Icarus 231, 34-50.

Goldreich, P., Tremaine, S. 1979. Precession of the epsilon ring of Uranus. Astron. J. 84, 1638-1641.

Graps, A. L., Showalter, M. R., Lissauer, J. J., Kary, D. M. 1995. Optical depths profiles and streamlines of the Uranian (epsilon) ring. Astron. J. 109, 2262-2273.

Gresh, D. L., Marouf, E. A., Tyler, G. L., Rosen, P. A., Simpson, R. A. 1989. Voyager radio occultation by Uranus' rings. I - Observational results. Icarus 78, 131-168.

Hahn, J. M., Spitale, J. N. 2013. An N-body integrator for gravitating planetary rings, and the outer edge of Saturn's B ring. Astrophys. J. 772, 122.

Hedman, M. M., Nicholson, P. D. 2013. Kronoseismology: Using density waves in Saturn's C ring to probe the planet's interior. Astron. J. 146, 12.

Hedman, M. M., Nicholson, P. D. 2014. More kronoseismology with Saturn's rings. Monthly Notices of the Royal Astron. Soc. 444, 1369-1388.

Jacobson, R. A., et al., 2006. The gravity field of the Saturnian system from satellite observations and spacecraft tracking data. Astron. J. 132, 2520-2526.

Marley, M. S. 1991. Nonradial oscillations of Saturn. Icarus 94, 420-435.

Marley, M. S. 2014. Saturn ring seismology: Looking beyond first order resonances. Icarus 234, 194-199.

Marley, M. S., Porco, C. C. 1993. Planetary acoustic mode seismology - Saturn's rings. Icarus 106, 508.

Marouf, E. A., Tyler, G. L., Zebker, H. A., Simpson, R. A., Eshleman, V. R. 1983. Particle size distributions in Saturn's rings from Voyager 1 radio occultation. Icarus 54, 189211.

Marouf, E. A., Gresh, D. L., Tyler, G. L. 1987. Eccentricity gradients of Uranus' epsilon, beta, and alpha rings. Bull. Am. Astron. Soc, 19, 883.

Mosqueira, I. 1996. Local simulations of perturbed dense planetary rings. Icarus 122, 128152.

Mosqueira, I., Estrada, P. R., Brookshaw, L. 1999. Hydrodynamical simulations of narrow planetary rings. I. Scaling. Icarus 139, 260-285.

Nicholson, P. D., et al., 2014a. Noncircular features in Saturn's rings: I. The edge of the B ring. Icarus 227, 152-175. 
Nicholson, P. D., French, R. G., McGhee-French, C. A., Hedman, M. M., Marouf, E. A., Colwell, J. E., Lonergan, K., Sepersky, T. 2014b. Noncircular features in Saturn's rings II: The $\mathrm{C}$ ring. Icarus 241, 373-396.

Porco, C. C. 1983. Voyager observations of Saturn's rings. 1: The eccentric rings at 1.29, 1.45, 1.95 and 2.27 RS. Ph.D. Thesis.

Porco, C. C., and 34 colleagues 2005. Cassini imaging science: Initial results on Saturn's rings and small satellites. Science 307, 1226-1236.

Porco, C., Nicholson, P. D., Borderies, N., Danielson, G. E., Goldreich, P., Holberg, J. B., Lane, A. L. 1984a. The eccentric Saturnian ringlets at $1.29 \mathrm{R}(\mathrm{s})$ and $1.45 \mathrm{R}(\mathrm{s})$. Icarus $60,1-16$.

Rein, H., Latter, H. N. 2013. Large-scale N-body simulations of the viscous overstability in Saturn's rings. Monthly Notices of the Royal Astron. Soc. 431, 145-158.

Shu, F. H. 1984. Waves in planetary rings. IAU Colloq. 75: Planetary Rings, Univ. of Arizona Press, 513-561.

Tiscareno, M. S., Burns, J. A., Nicholson, P. D., Hedman, M. M., Porco, C. C. 2007. Cassini imaging of Saturn's rings. II. A wavelet technique for analysis of density waves and other radial structure in the rings. Icarus 189, 14-34.

This preprint was prepared with the AAS LATEX macros v5.2. 


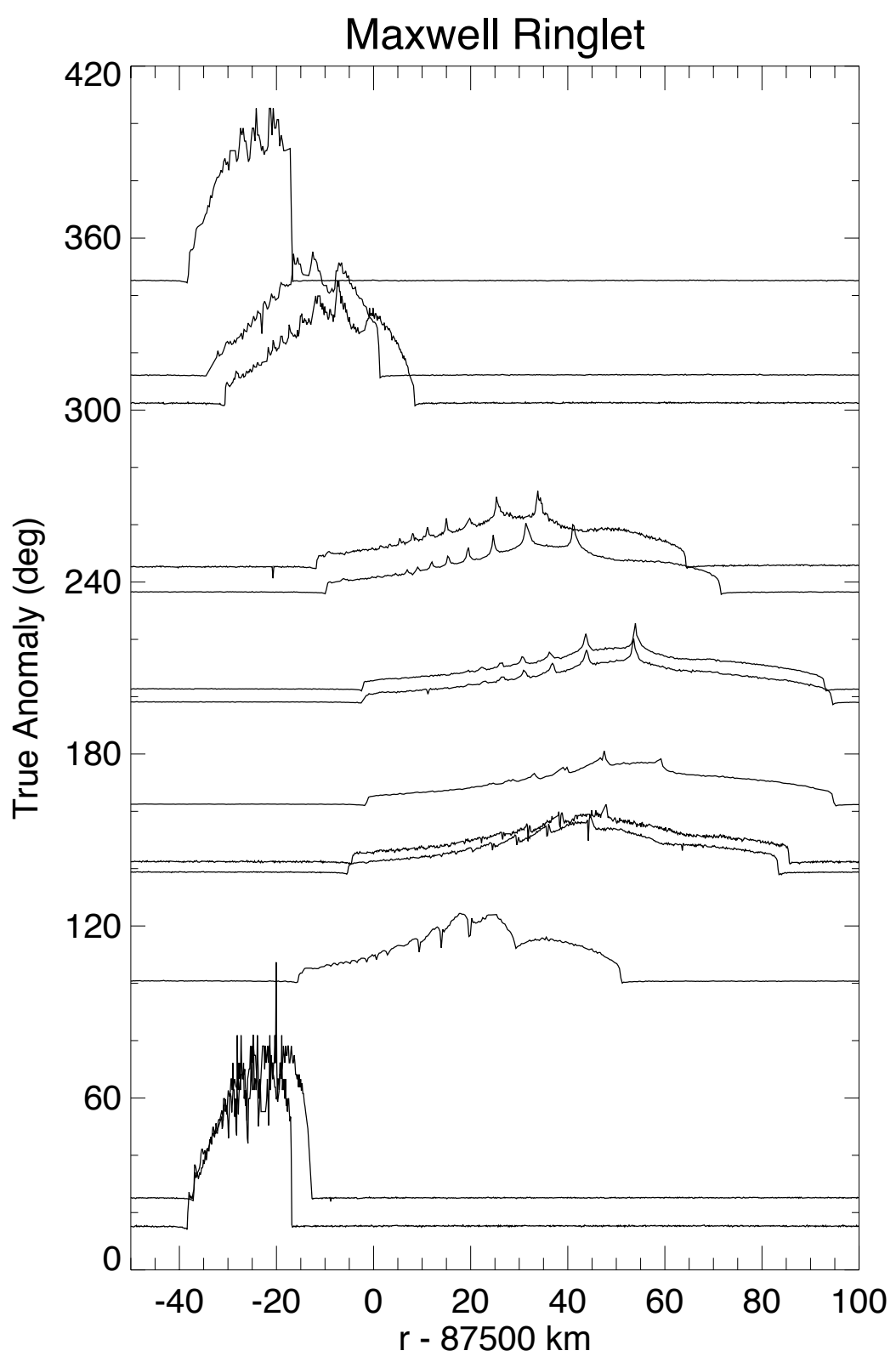

(wellRinglet_v2.pro/Users/frrench/Research/RINGFIT/tests/Saturn/Sa025S/programs/../figs/plot_optical_depth_scans_MaxwellRinglet_v2_20150208a.ps

Fig. 1. - A sequence of optical depth profiles of the Maxwell ringlet derived from 13 Cassini VIMS $\gamma$ Cru occultations, offset vertically by true anomaly. Note the wavelike internal structure in the inner two-thirds of the ringlet. 


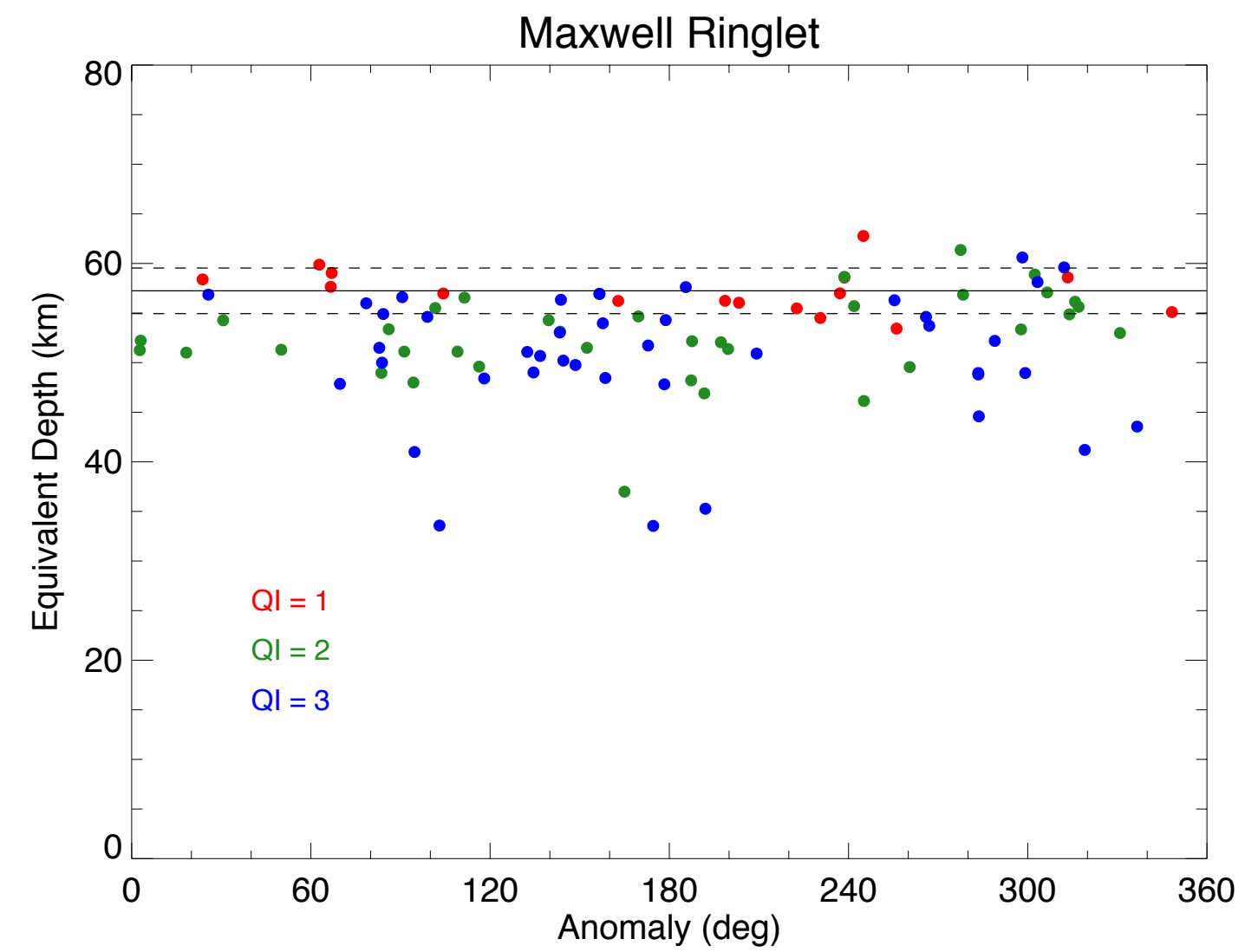

rfrench@Achilles.home Sat Jan 31 11:10:40 2015 eqdepth_maxwell_20150105.pro /Users/frrench/Research/RM/RINGMASTER/programs/../figs/eqdepth_maxwell_20150131a.ps

Fig. 2. - The radially-integrated optical depth (or equivalent depth) of the Maxwell ringlet in our better-quality profiles. The observations are color-coded by quality index (QI), as indicated. The solid horizontal line marks the mean equivalent depth of the $\mathrm{QI}=1$ points: $A=57.24 \pm 2.30 \mathrm{~km}$, with dashed lines showing its uncertainty. There appears to be no systematic variation with true anomaly, but lower-SNR profiles give lower values, probably because of saturation in the estimates of the optical depth of the wave peaks. 


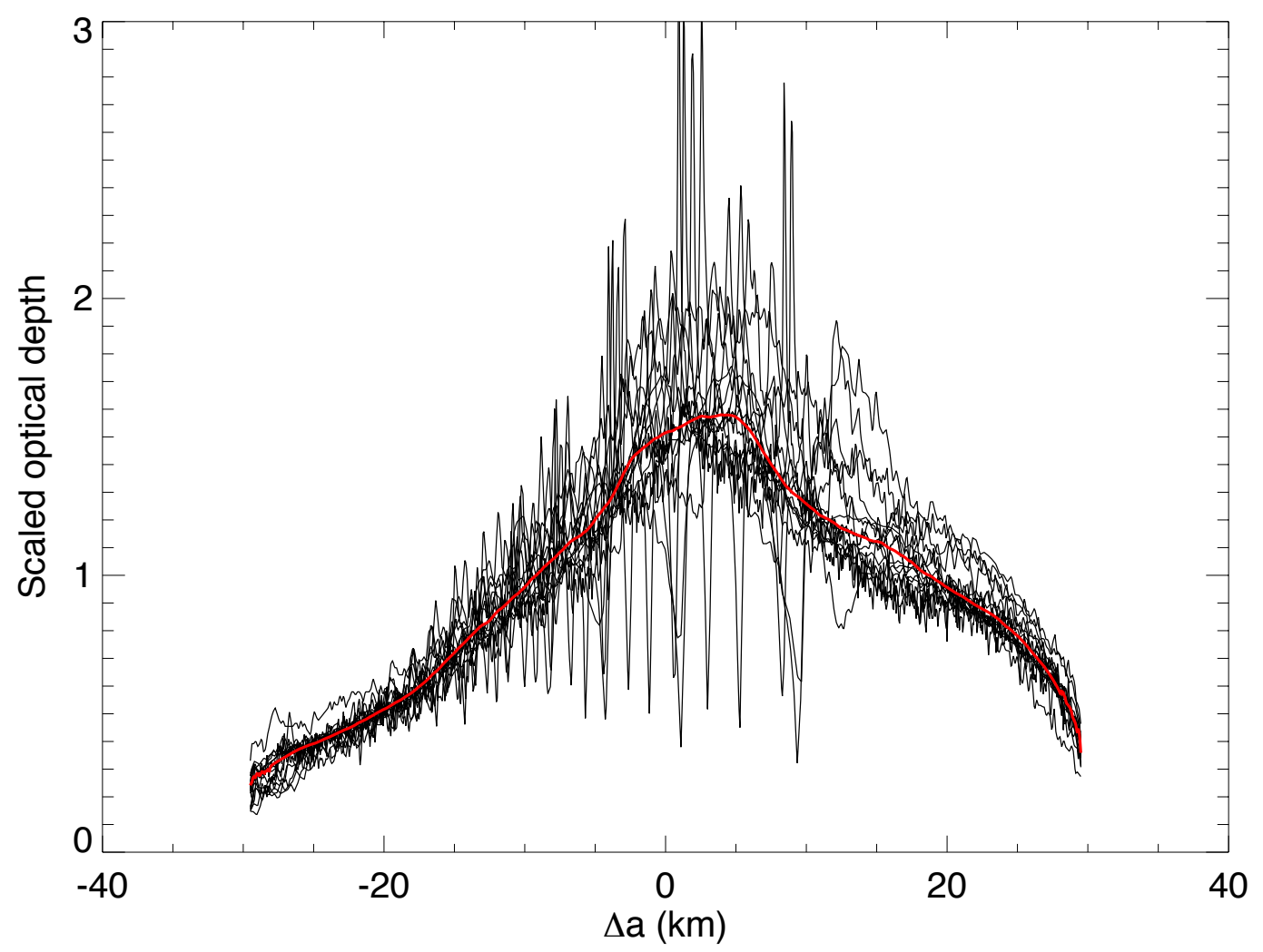

Fig. 3.- The $16 \mathrm{QI}=1$ profiles, rescaled to a mean width of $59.1 \mathrm{~km}$, together with the mean optical depth profile in red. The 1000-point profiles have been smoothed by 50 bins $(3.0 \mathrm{~km})$. The smoothed mean profile has an equivalent depth of $56.48 \mathrm{~km}$, compared to the mean value in Fig. 2 of $57.24 \mathrm{~km}$, or a difference of $\sim 0.33 \sigma$. 

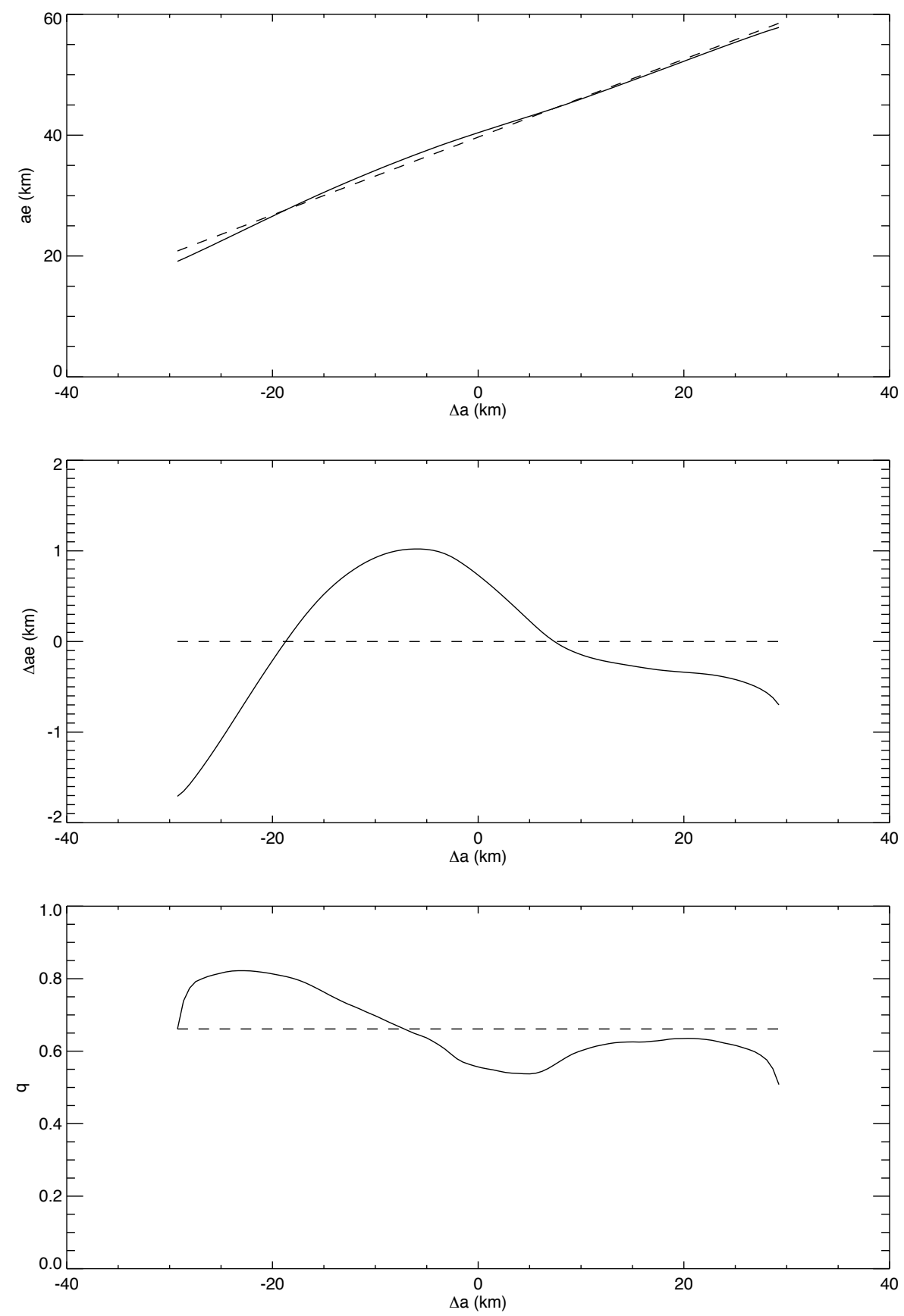

Fig. 4.- Radial eccentricity profile $a e(\Delta a)$ derived from the self-gravity model (solid line) compared to a linear eccentricity model (dashed line). The middle panel shows the difference between the self-gravity and linear models, and the lower panel shows the self-gravity eccentricity gradient profile $q(a)=a d e / d a$, compared to the mean value for the linear model of $\bar{q}=0.66$. 


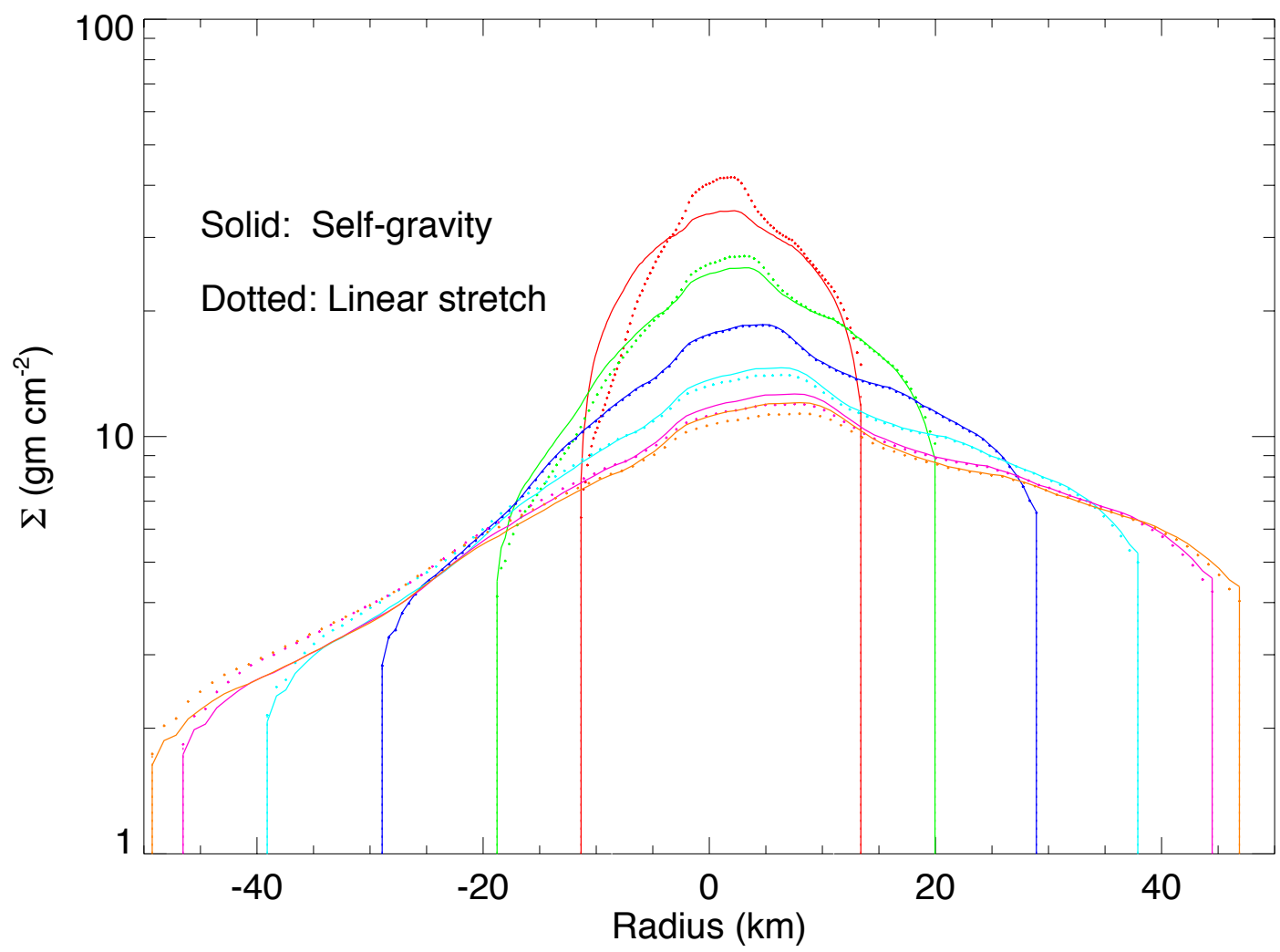

rfrench@Achilles.home Sat Jan 31 11:02:09 2015 GTselfgrav.pro /Users/ffrench/Research/RM/RINGMASTER/selfgrav/programs/./figs/GTselfgrav_20150131a.ps

Fig. 5.- Optical depth profiles for the Maxwell ringlet derived from the self-gravity model (solid lines) compared with those from the simple linear-stretch model (dotted lines) used to rescale the observations in Fig. 12. Different colors denote different values of the true anomaly. By design, the profiles match at quadrature (blue curves). The average surface mass density at quadrature is about $11 \mathrm{~g} \mathrm{~cm}^{-2}$, as derived from the self-gravity model. Note the differences in shape of the profiles near periapse (red curves) and apoapse (orange and magenta curves). 

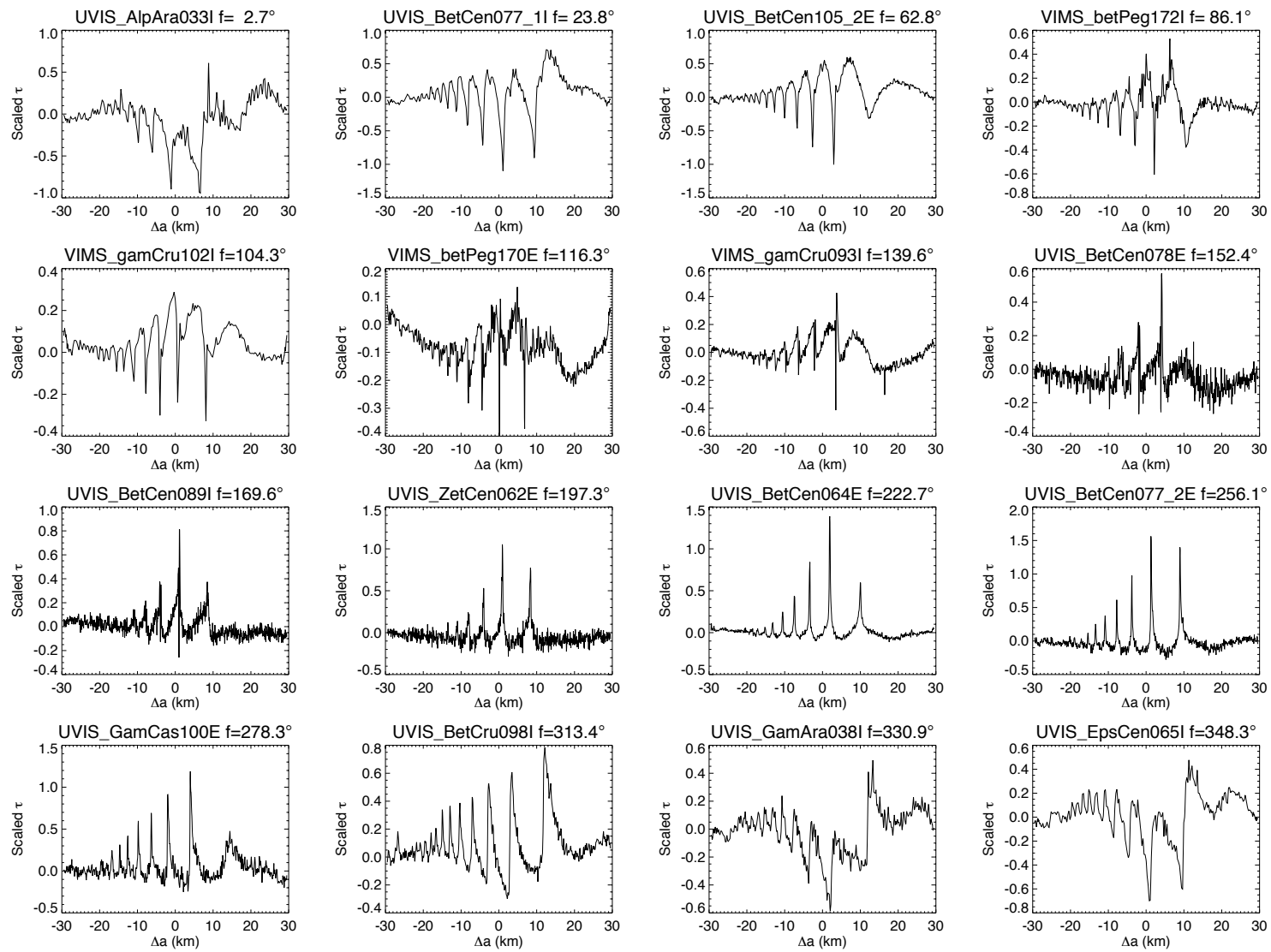

rfrench@Achilles.home Tue Jan 6 17:46:36 2015 bkg_subtracted_maxwell_20150105.pro /Users/frrench/Research/RM/RINGMASTER/programs/../figs/bkg_subtracted_maxwell_20150106a.ps

Fig. 6.- Rescaled and background-subtracted optical depth profiles of the Maxwell ringlet, sorted by true anomaly. Labels indicate the instrument, star name, Cassini rev number, and true anomaly $f$ for each profile. These are selected from $52 \mathrm{QI}=1$ and QI $=2$ profiles to illustrate the full range of variation seen in the wave profiles. Note the transitions in profile shape near $f \sim 135^{\circ}$ and $\sim 330^{\circ}$. 


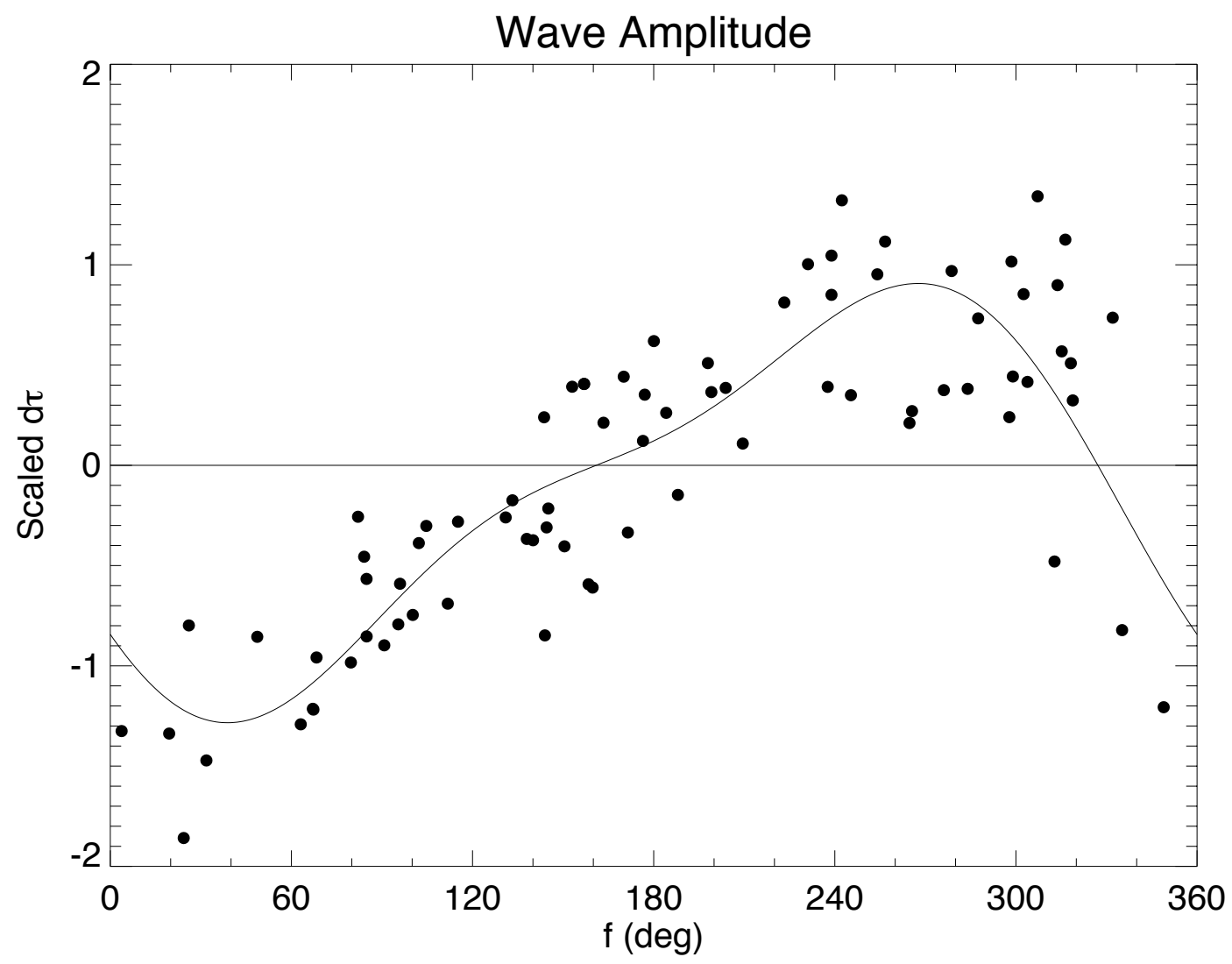

rfrench@Achilles.home Tue Jan 6 19:21:24 2015 MaxwellRinglet_fit_spike.pro /Users/ffrench/Research/RM/RINGMASTER/programs/../figs/MaxwellRinglet_fit_spike.20150106a.ps

Fig. 7.- Variation of peak amplitude of crests or troughs for the central part of the wave vs true anomaly, using all profiles with $\mathrm{QI}=1,2$ or 3 . The curve is a third-order Fourier fit. See text for details. 


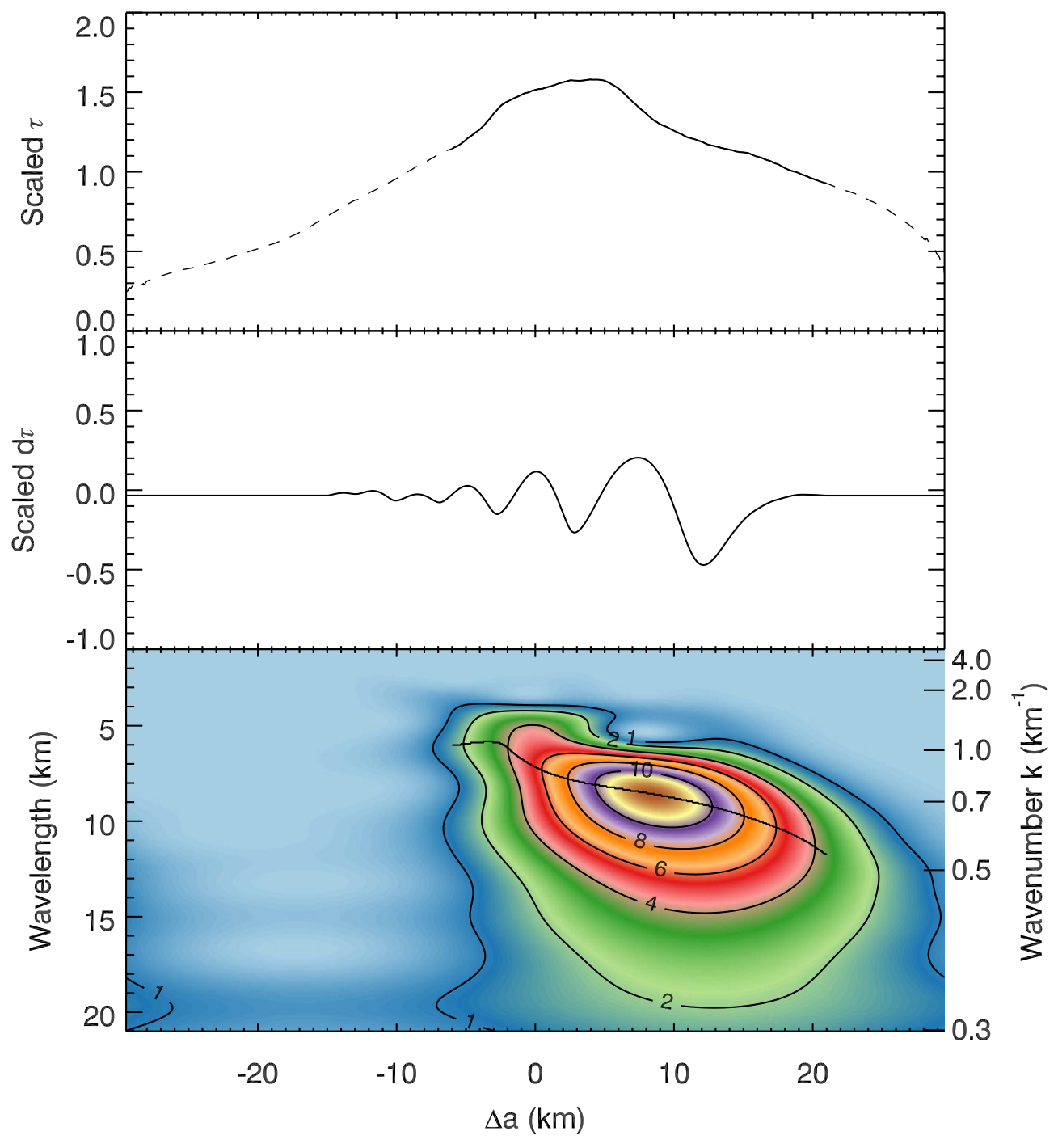

Fig. 8.- An illustration of the wavelet decomposition procedure. The top panel shows the mean optical depth profile, the middle panel shows a representative smoothed backgroundsubtracted optical depth profile, rescaled as in Fig. 6, while the bottom panel shows a contour map of the wavelet power vs rescaled radius and wavelength (left axis) and radial wavenumber $k(r)$ (right axis). The resonant radius for the wave is estimated to be $87,530 \mathrm{~km}$, or $\Delta a=+20 \mathrm{~km}$. 

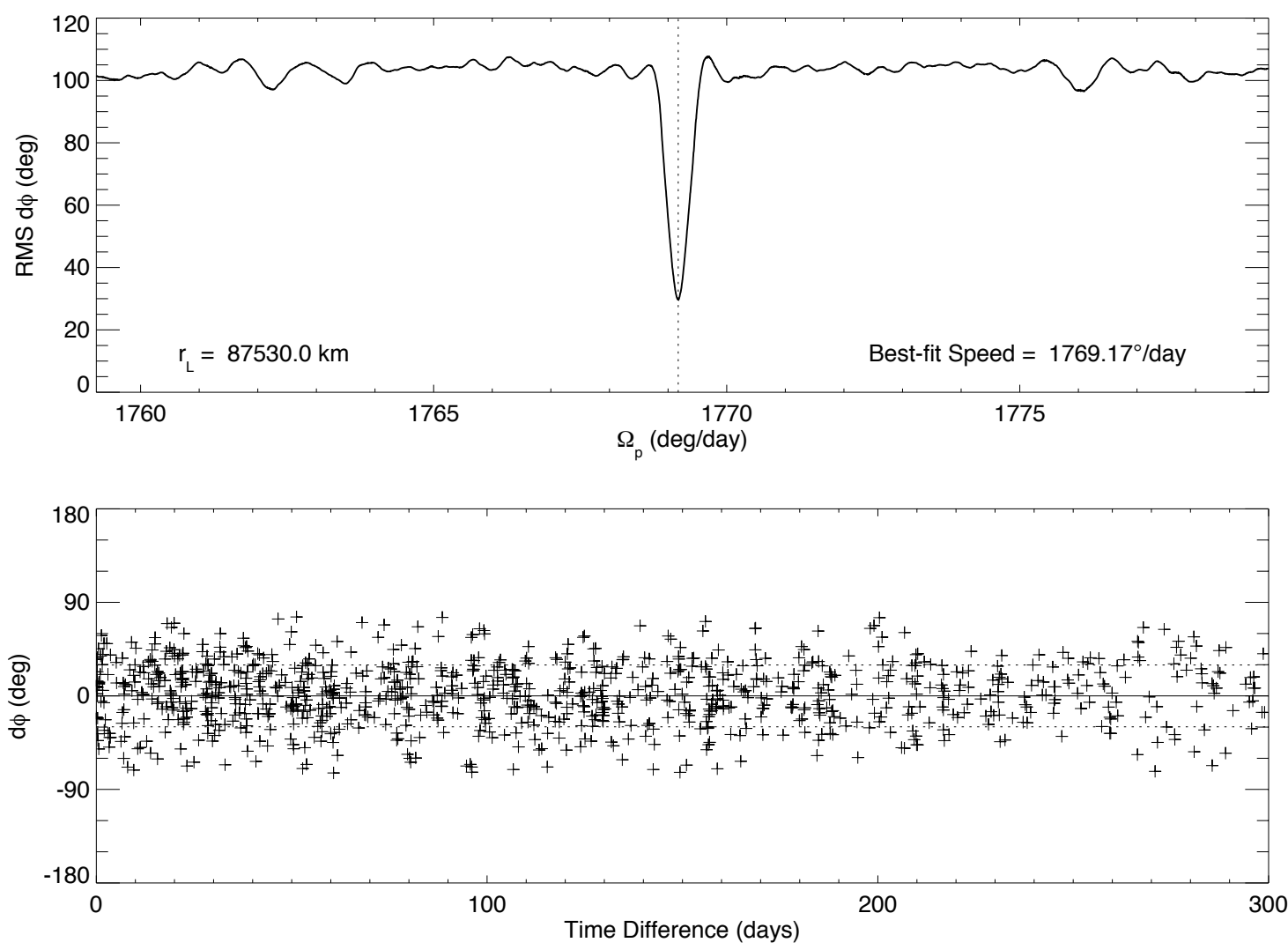

ffrench@Achilles.home Sun Feb 8 15:12:29 2015 MaxwellRinglet_period_search_v4.pro /Users/frrench/Research/RM/RINGMASTER/programs/../figs/MaxwellRinglet_period_search_20150208a.ps

Fig. 9.- Periodogram showing the detection of an $m=-2$ OLR-type wave in the Maxwell ringlet. The minimum in the RMS phase residuals occurs for a pattern speed $\Omega_{p}=1769.17^{\circ} \mathrm{d}^{-1}$, corresponding to $a_{\text {res }}=87,530.0 \mathrm{~km}$. The lower panel shows all 1059 individual phase residuals, $\delta \phi_{\text {obs }}-\delta \phi_{\text {pred }}$, as a function of the time interval between observations $\delta t$. 


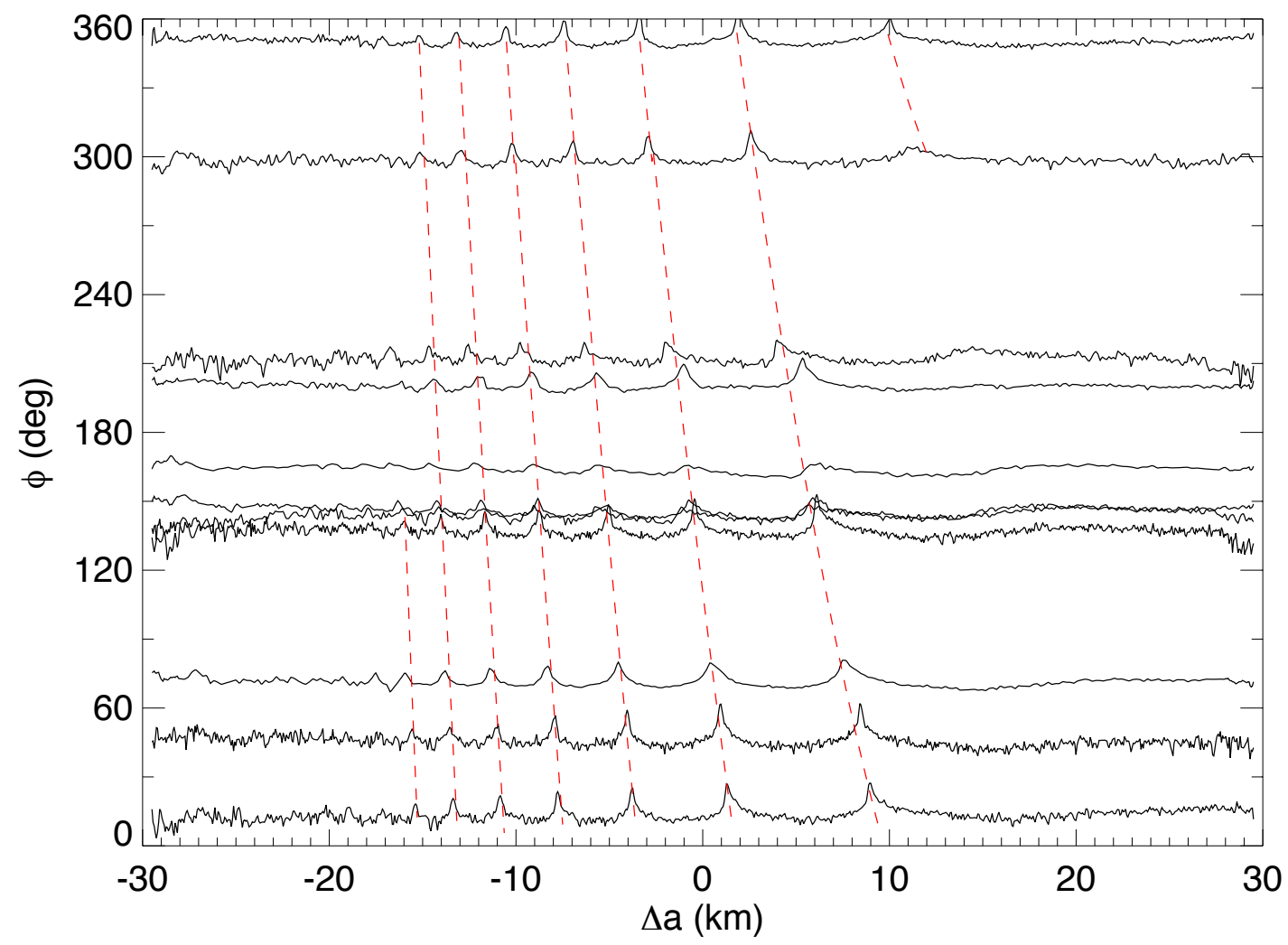

rfrench@Achilles.home Sat Jan 31 11:05:54 2015 Maxwell_trailing_v2.pro /Users/ffrench/Research/RM/RINGMASTER/programs/..figs/Maxwell_trailing_v2_20150131a.ps

Fig. 10.- A series of rescaled, background-subtracted wave profiles in the range of true anomaly $200^{\circ}<f<300^{\circ}$, sorted by, and offset vertically in proportion to, the wave phase $\phi=|m|\left(\lambda-\Omega_{p} t\right)$. We have set $m=-2$ and assumed the best-fit pattern speed from Fig. 9 . The trailing spiral nature of the pattern is evident (see text), as is its 2-armed nature. The dashed curve is a line of constant three-dimensional phase, $\phi(\Delta a, \lambda, t)$, as given by Eq. (3), using the empirical radial phase function $\phi_{r}(\Delta a)$ derived in Section 4.4 

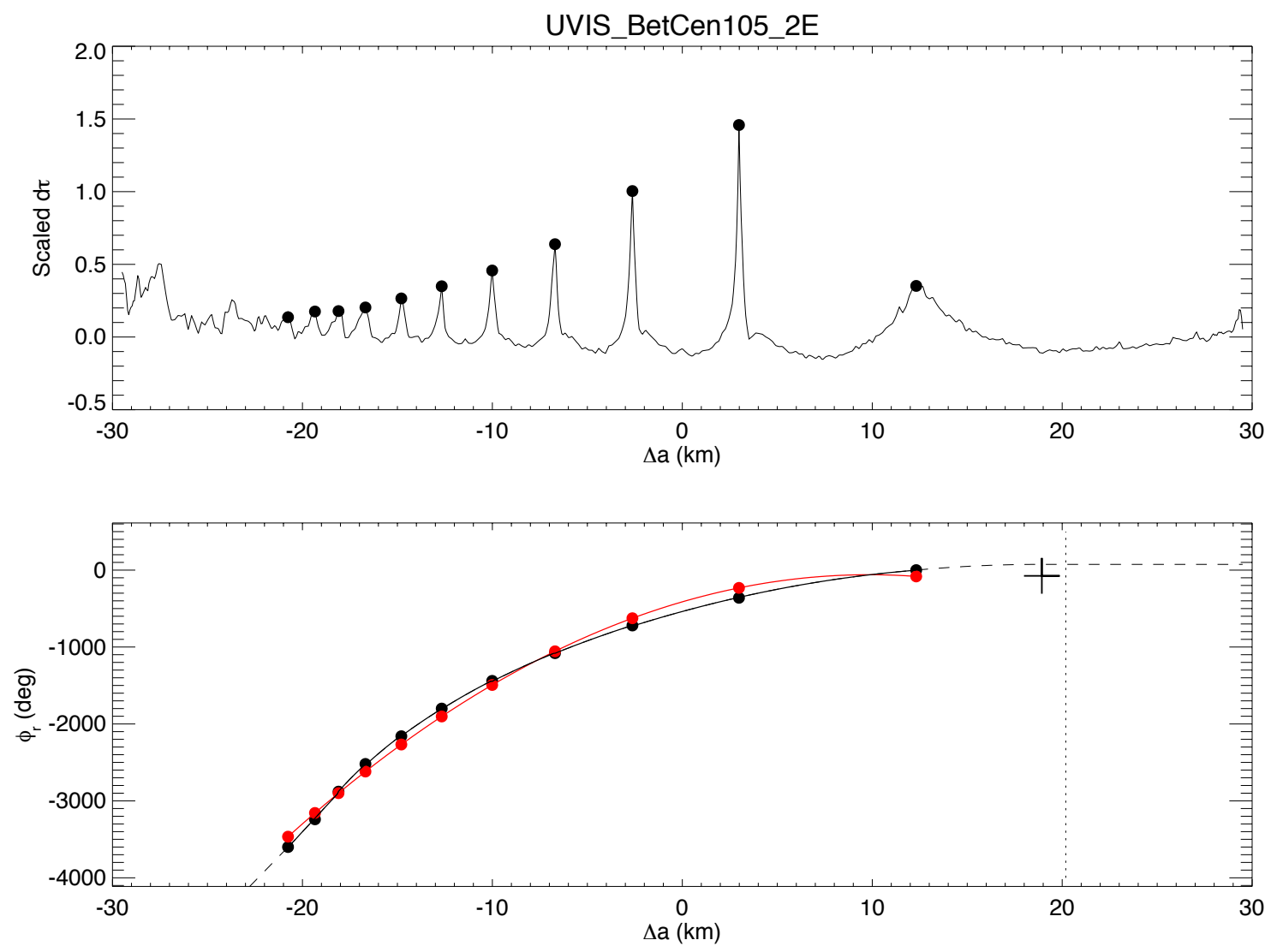

rfrench@Achilles.home Sat Jan 10 17:30:55 2015 Maxwell_estimate_q.pro /Users/frrench/Research/RM/RINGMASTER/programs/../figs/Maxwell_estimate_q_20150110a.ps

Fig. 11. - Determination of radial wave phase $\phi_{r}$ as a function of rescaled radius $\Delta a$. The top panel shows a rescaled, background-subtracted occultation profile, with the successive wave crests identified, while the bottom panel shows the corresponding cumulative phase, starting from zero at the inferred resonant radius, $\Delta a_{\text {res }}=+18.9 \mathrm{~km}$. The red curve is a simple quadratic model fitted to the reconstructed phase profile (see text). The vertical dotted line indicates the resonance radius derived from the phase residuals in Fig. 9, while the + symbol marks the radius at which the quadratically-extrapolated empirical $\phi_{r}$ has zero slope. Note the close agreement between these independent estimates of the resonance radius. 

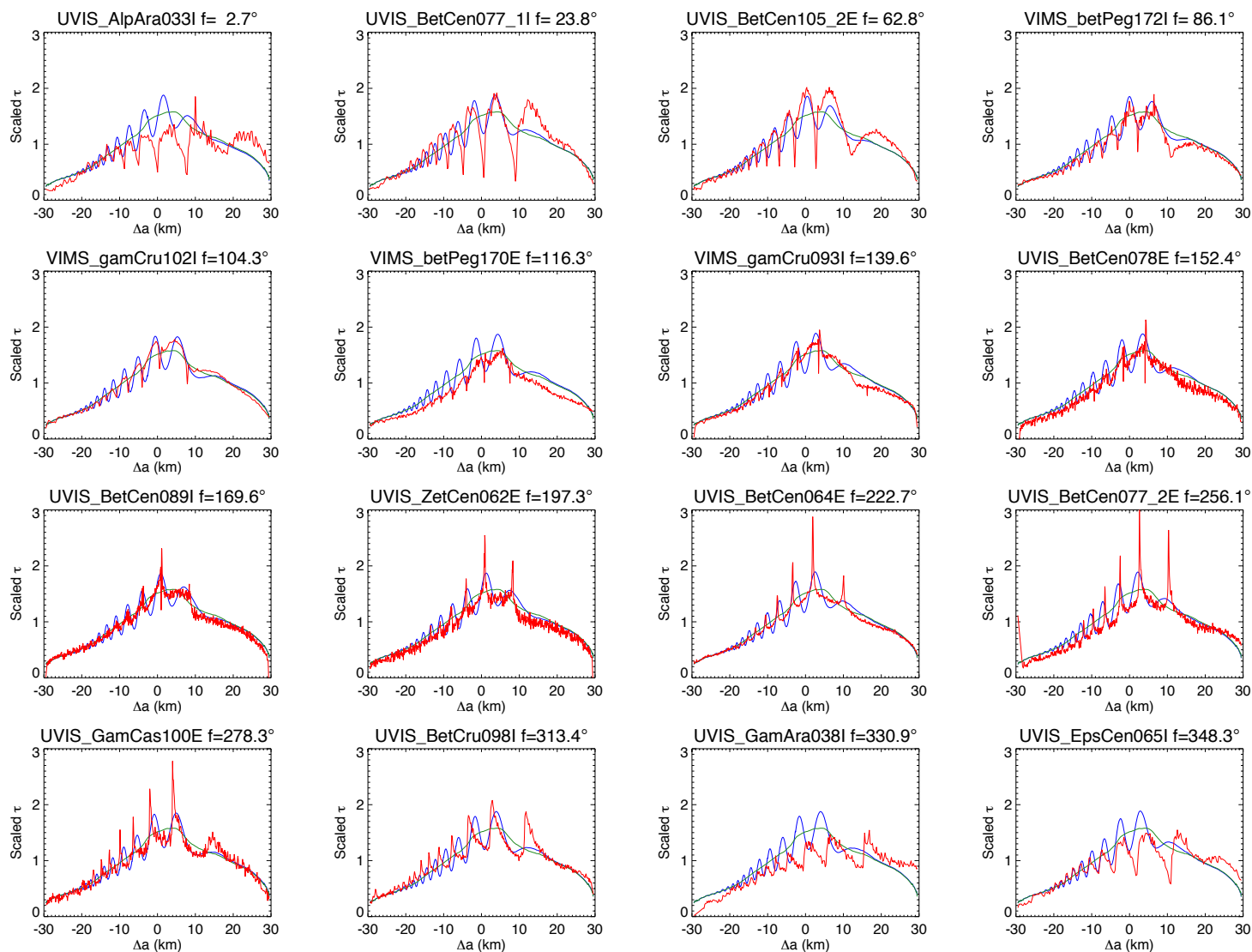

rfrench@Achilles.home Tue Jan 6 17:27:33 2015 Maxwell_taumodel_v4.pro /Users/frrench/Research/RM/RINGMASTER/programs/../figs/ps/Maxwell_taumodel_v4_20150106d.ps

Fig. 12.- Comparison of the linear density wave model with observations. The 16 Cassini VIMS or UVIS occultation profiles used in Fig. 6 are shown in order of increasing true anomaly $f$, as labeled in the title of each panel. The rescaled optical depths are shown in red; the blue curves show the profiles predicted by the linear density wave model; and the green curve is the mean background profile $\tau_{0}(\Delta a)$. No free parameters have been adjusted for any individual profile, beyond the common values of $A_{L}, \xi_{D}$ and $\phi_{0}$. The phase of the wave is calculated from its known pattern speed, the inertial longitude of the occultation profile, and the time of the observation. 


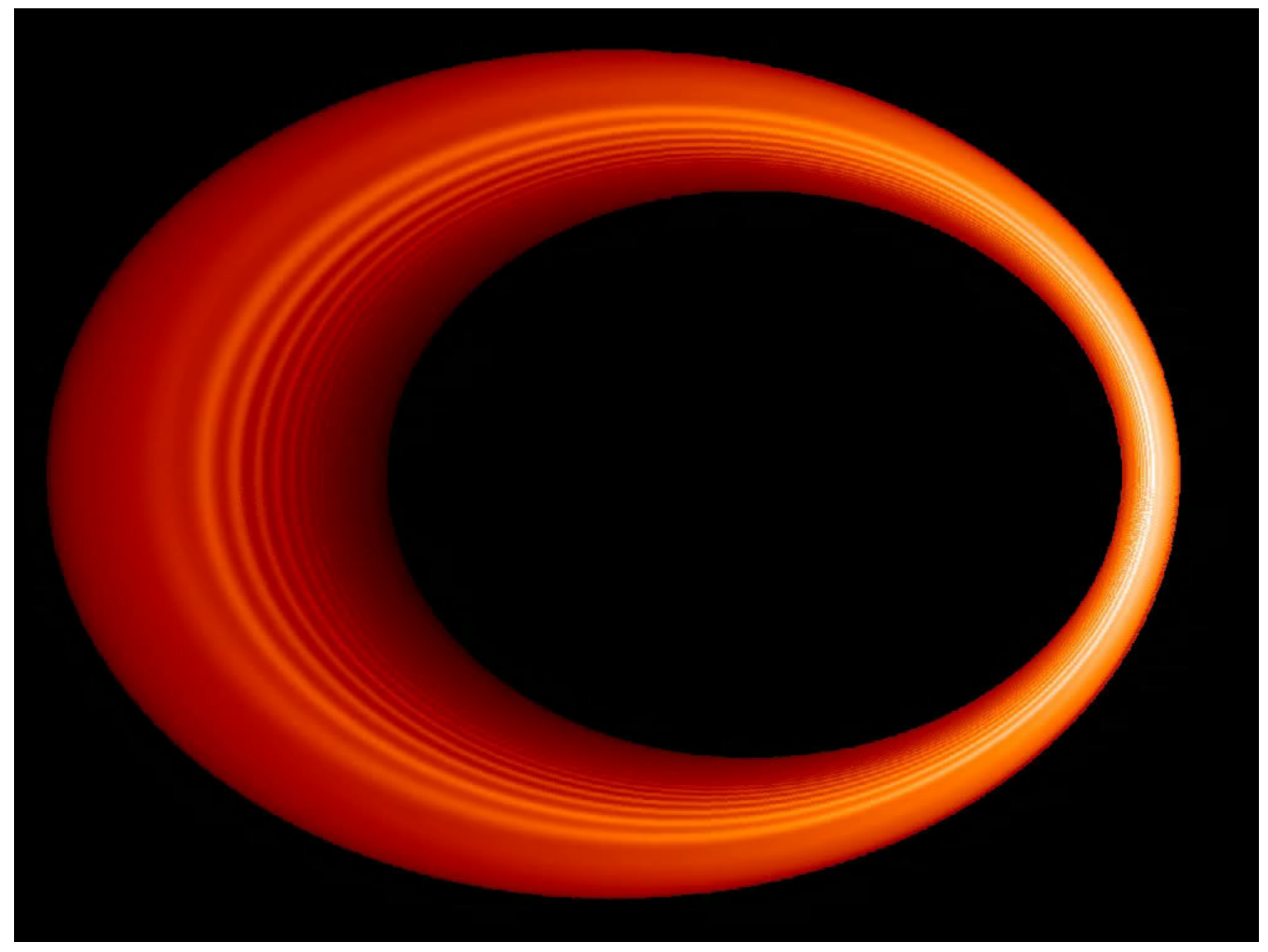

Fig. 13.- A graphical depiction of the $m=-2$ spiral wave structure in the Maxwell ringlet, based on a linear density wave model and a simple linear-stretch model for the background optical depth profile. The ringlet's radial width and eccentricity are exaggerated for clarity, but the relative radial scales of the wave and ring and the fractional variation in ring width are all accurate. Brightness is proportional to optical depth. 


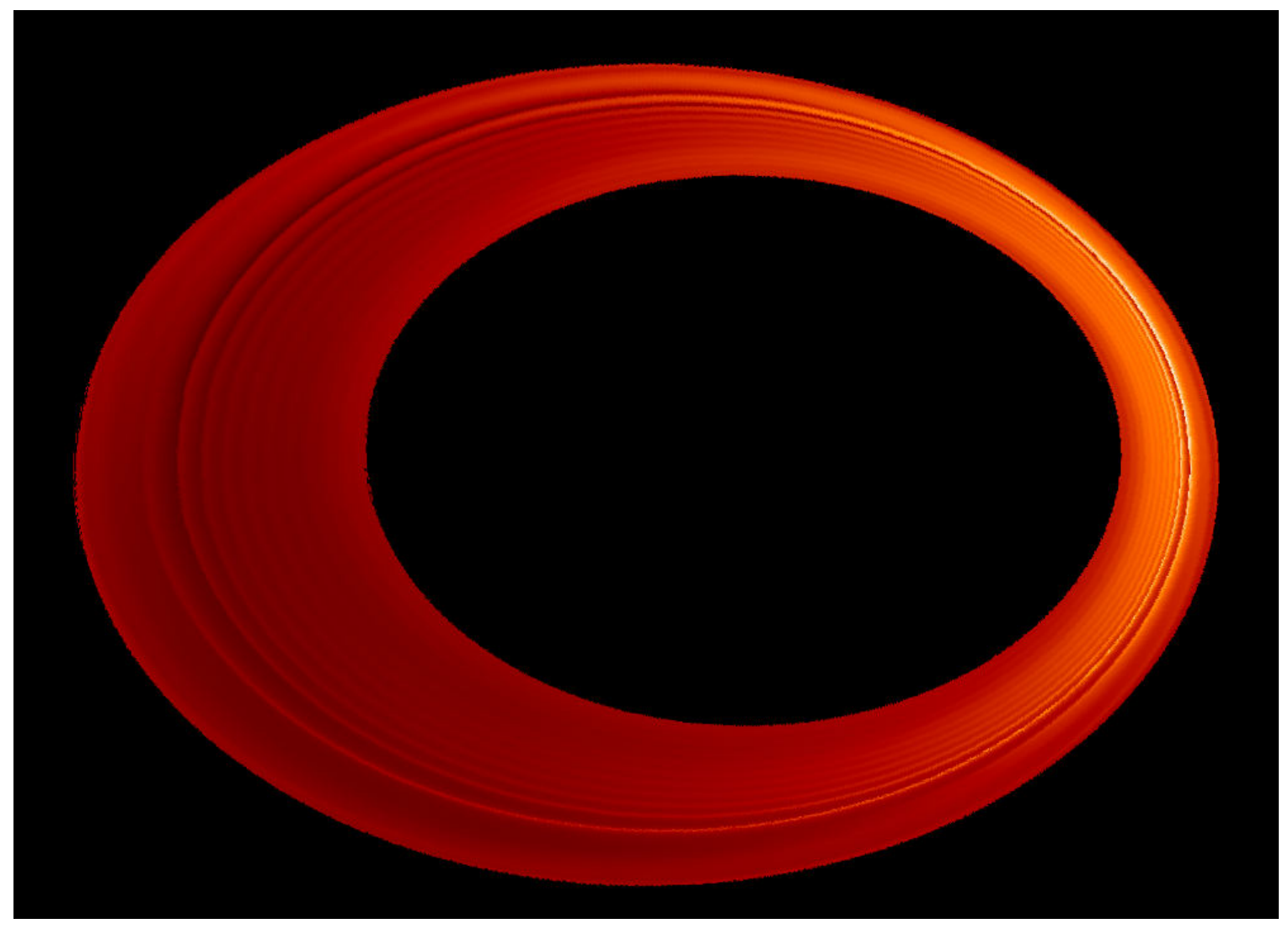

Fig. 14. - Symplectic N-body simulation of an $m=-2$ wave developed within an eccentric ringlet, due to resonant forcing by a hypothetical interior satellite. The ringlet's radial width and eccentricity are exaggerated for clarity, but the relative radial scales of the wave and ring and the fractional variation in ring width are preserved from the model values. Compare the wave structure resulting from this numerical integration with that of the linear density wave model for the Maxwell ringlet in Fig. 13. 

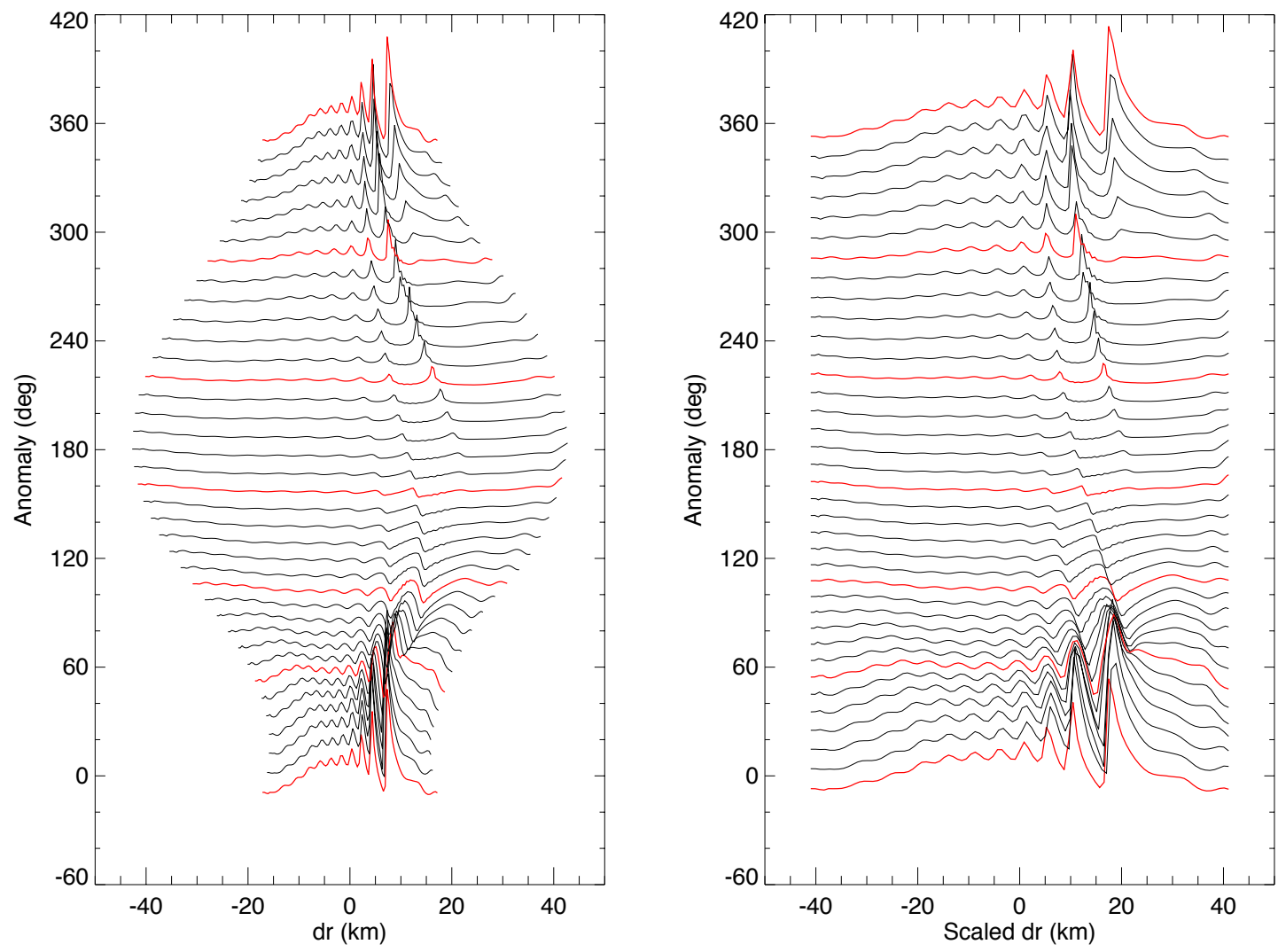

ench@Achilles.home Tue Jun 16 19:52:31 2015 plot_fangs_cas0139.030000.pro /Users/french/Research/RM/RINGMASTER/epi_int_rgf/programs/../figs/plot_fangs_cas0139.030000_v2.20150616b.ps

Fig. 15.- Optical depth profiles across the simulated ringlet in Fig. 14, plotted at intervals of $10^{\circ}$ in true anomaly, showing the development of an $m=-2$ OLR-type wave. The left panel shows the raw profiles in real radius and the right panel the rescaled profiles, for comparison with the observations in Fig. 6. (In this case, the profiles have been rescaled horizontally to the maximum ring width, assumed to be $80 \mathrm{~km}$, rather than the mean width, and scaled vertically to preserve the radially integrated optical depth of each profile.) The main qualitative characteristics of the observed wave are reproduced here, including the transitions between sharp peaks and sharp troughs. Note the strong compression of the wave profile near periapse. Profiles are shown in red at intervals of $60^{\circ}$ in true anomaly, for ease of comparison with individual occultation profiles observed at nearby anomalies, in Fig. 6. 

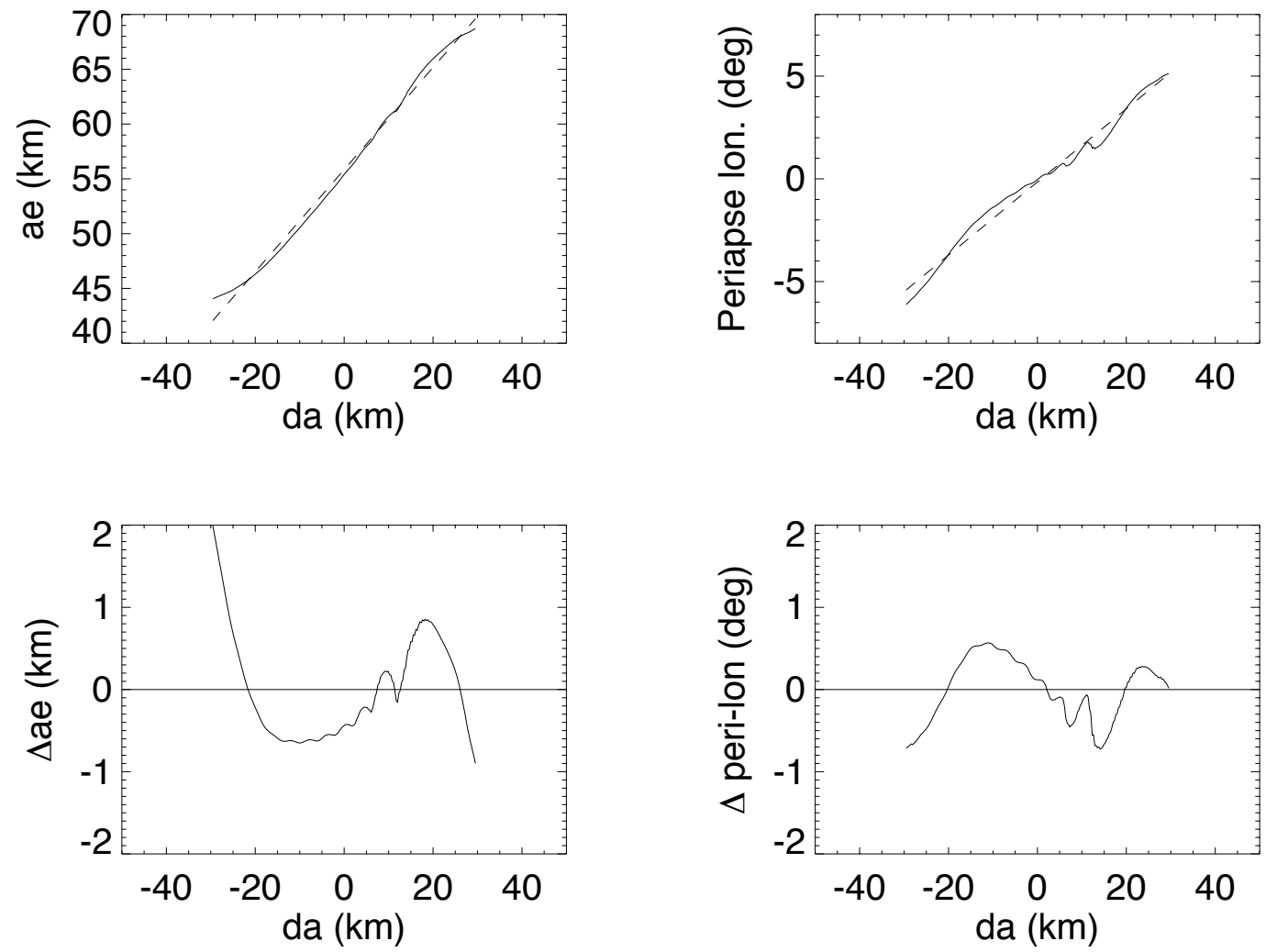

rfrench@phoebe Thu Jun 4 14:39:31 2015 plot_ecc_anom.pro Nolumes/dione_raid2/Research/RM/RINGMASTER/epi_int_rgf/programs/epi_int_0139_ecc+anom_20150604a.ps

Fig. 16.- Radial variations in streamline eccentricity and periapse longitude, from our standard epi_int simulation. The upper left panel compares the eccentricity profile at the conclusion of the integration to the initial linear model, shown as a dashed line. The lower left panel shows the difference between these two profiles, revealing periodic structure associated with the internal density wave that has developed over the course of the simulation, superimposed on a more regional quasi-sinusoidal residual pattern. At upper right, the periapse longitude is shown to vary roughly linearly with orbital radius; the difference between the actual variation and a linear approximation is shown in the lower right panel. Again, the wavelike appearance reflects the structure of the internal wave generated in the simulation by a fictitious satellite. The combination of periodic variations in the eccentricity profile and the periapse longitude of the particle streamlines results in the optical depth profiles shown in Fig. 15. 


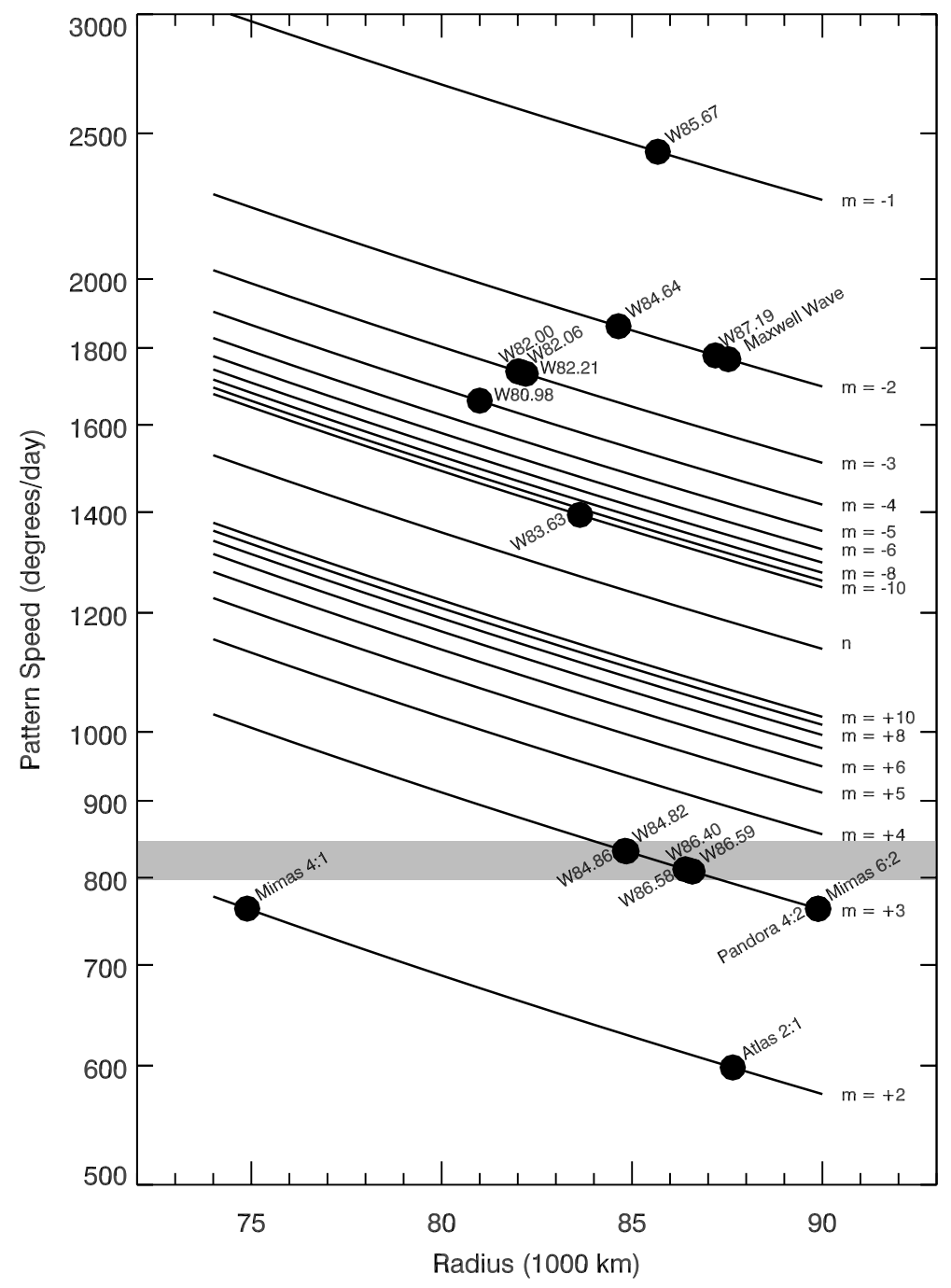

Fig. 17.- Resonance locations for waves driven in Saturn's C ring. The curves indicate the predicted pattern speeds for OLR-type $(m<0)$ and ILR-type $(m>0)$ waves as functions of orbital radius, while filled circles denote satellite-driven and internal-mode-driven waves identified to date. Labels identify the specific waves. The outermost $m=-2$ wave is the one in the Maxwell ringlet. Note that there are now three identified waves for $m=-2$ and three for $m=-3$, but only single waves for $m=-1, m=-4$ and $m=-10$. The grey band indicates the observed range of Saturnian rotation rates. (Figure modified from Hedman and Nicholson (2014).) 